\title{
Graphs and complete intersection toric ideals
}

\author{
I. Bermejo ${ }^{\mathrm{a}, 1, *}$, I. García-Marco ${ }^{\mathrm{a}, 1}$, E. Reyes ${ }^{\mathrm{b}, 2}$ \\ ${ }^{a}$ Facultad de Matemáticas, Universidad de La Laguna, 38200 La Laguna, Tenerife, Canary Islands, Spain \\ ${ }^{b}$ Departamento de Matemáticas, Centro de Investigación y de Estudios Avanzados del IPN, Apartado Postal 14-740, 0700 México City, D.F., \\ Mexico
}

\begin{abstract}
Our purpose is to study the family of simple undirected graphs whose toric ideal is a complete intersection from both an algorithmic and a combinatorial point of view. We obtain a polynomial time algorithm that, given a graph $G$, checks whether its toric ideal $P_{G}$ is a complete intersection or not. Whenever $P_{G}$ is a complete intersection, the algorithm also returns a minimal set of generators of $P_{G}$. Moreover, we prove that if $G$ is a connected graph and $P_{G}$ is a complete intersection, then there exist two induced subgraphs $R$ and $C$ of $G$ such that the vertex set $V(G)$ of $G$ is the disjoint union of $V(R)$ and $V(C)$, where $R$ is a bipartite ring graph and $C$ is either the empty graph, an odd primitive cycle, or consists of two odd primitive cycles properly connected. Finally, if $R$ is 2 -connected and $C$ is connected, we list the families of graphs whose toric ideals are complete intersection.
\end{abstract}

Keywords: homogeneous toric ideal, graph, complete intersection

2010 MSC: 14M25, 05C25, 05E40

\section{Introduction}

Let $k$ be an arbitrary field and $A=\left(a_{i j}\right)$ an $m \times n$ matrix with non negative integer entries $a_{i j}$ and with non-zero columns. Let $k\left[x_{1}, \ldots, x_{n}\right]$ and $k\left[t_{1}, \ldots, t_{m}\right]$ be two polynomial rings over $k$. Denote by $x^{b}$ the monomial $x_{1}^{b_{1}} \cdots x_{n}^{b_{n}}$, where $b=\left(b_{1}, \ldots, b_{n}\right) \in \mathbb{N}^{n}$. A binomial $f$ in $k\left[x_{1}, \ldots, x_{n}\right]$ is a difference of two monomials, i.e., $f=x^{b}-x^{c}$ for some $b, c \in \mathbb{N}^{n}$. An ideal generated by binomials is called a binomial ideal. Consider $\varphi$ the graded homomorphism of $k$-algebras

$$
\varphi: k\left[x_{1}, \ldots, x_{n}\right] \rightarrow k\left[t_{1}, \ldots, t_{m}\right] \text { induced by } \varphi\left(x_{i}\right)=t^{a_{i}},
$$

where $a_{i}$ is the $i$-th column of $A$. The polynomial rings are graded by assigning $\operatorname{deg}\left(t_{i}\right)=1$ and $\operatorname{deg}\left(x_{j}\right)=\operatorname{deg}\left(t^{a_{j}}\right)$ for every $i, j$. The kernel of $\varphi$, denoted by $P_{A}$, is called the toric ideal associated to $A$. It is well-known that $P_{A}$ is a prime graded binomial ideal with ht $\left(P_{A}\right)=n-\operatorname{rank}(A)$ (see for example [27, 30]).

$P_{A}$ is a complete intersection if $\mu\left(P_{A}\right)=\operatorname{ht}\left(P_{A}\right)$, where $\mu\left(P_{A}\right)$ denotes the minimal number of generators of $P_{A}$. Equivalently, $P_{A}$ is a complete intersection if and only if there exists a set of homogeneous binomials $f_{1}, \ldots, f_{r} \in$ $k\left[x_{1}, \ldots, x_{n}\right]$ such that $r=\mathrm{ht}\left(P_{A}\right)$ and $P_{A}=\left(f_{1}, \ldots, f_{r}\right)$.

Complete intersection toric ideals were first studied by Herzog in [14]. After that, they have been extensively studied by several authors; see for example [2, 3, 20] and the references there. It is well known, see e.g. [6] or [24], that the problem of deciding whether a toric ideal is a complete intersection belongs to the complexity class $\mathcal{N} \mathcal{P}$.

${ }^{*}$ Corresponding author. Tel: +34922318161

Email addresses: ibermejo@ull.es (I. Bermejo), iggarcia@ull.es (I. García-Marco), ereyes@math.cinvestav.mx (E. Reyes)

${ }^{1}$ Partially supported by Ministerio de Ciencia e Innovación, Spain (MTM2010-20279-C02-02).

${ }^{2}$ Partially supported by SNI, Mexico. 


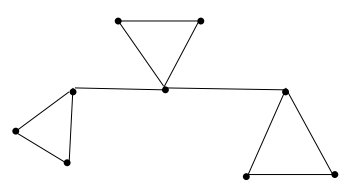

Figure 1: Ring graph which is not a complete intersection

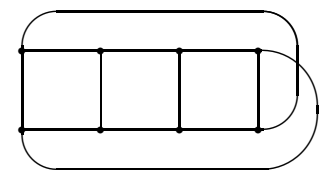

Figure 2: Non planar graph which is a complete intersection

Let $G$ be a simple undirected graph, i.e., an undirected graph without multiple edges or loops. Set $V(G)=$ $\left\{v_{1}, \ldots, v_{m}\right\}$ its vertex set, $E(G)=\left\{e_{1}, \ldots, e_{n}\right\}$ its edge set, and $A_{G}$ its incidence matrix. The toric ideal associated to $A_{G}$ is denoted by $P_{G}$. It is a prime homogeneous binomial ideal called the toric ideal of $G$. The image of $\varphi$ is denoted by $k[G]$ and called the edge algebra of $G$. If we denote by $b(G)$ the number of connected components of $G$ which are bipartite, then $\operatorname{rank}\left(A_{G}\right)=m-b(G)$ (see [31]) which implies that ht $\left(P_{G}\right)=n-m+b(G)$. We say that $G$ is a complete intersection if the corresponding toric ideal $P_{G}$ is a complete intersection.

In this work we study the complete intersection property of graphs from both an algorithmic and a combinatorial point of view.

The complete intersection property for bipartite graphs has been extensively studied; see for example $[5,10$, 11, 12, 17, 25]. It is worth mentioning that Gitler, Reyes and Villarreal proved in [11] that a bipartite graph is a complete intersection if and only if it is a ring graph. Since ring graphs are obviously planar, they could derive that every complete intersection bipartite graph is planar, which was previously proved by Katzman [17] without using the notion of ring graph. When graphs are not necessarily bipartite there is some recent work by Tatakis and Thoma [28], in the last section we make use of some of their technical results. For directed graphs, the complete intersection property has also been widely studied, see for example [9, 11, 22].

In this work, our graphs are undirected and not necessarily bipartite. In this general setting, the problem requires a different approach. Indeed, Figure 1 shows an example of a ring graph whose toric ideal is not a complete intersection. Moreover, there exist complete intersection graphs which are not ring graphs; Figure 2 shows a complete intersection graph which is not even planar.

The main results of this work are Theorem 4.8 , Theorem 6.5 and Theorem 6.18, The first one yields a polynomial time algorithm which receives as input a simple undirected graph $G$ and returns TRUE if $G$ is a complete intersection or FaLSE otherwise. Moreover, whenever $G$ is a complete intersection, the algorithm provides without any extra effort a minimal set of generators of $P_{G}$. As a consequence of this algorithm we obtain that the problem of determining whether a graph $G$ is a complete intersection belongs to the complexity class $\mathcal{P}$. Given a connected graph $G$, we get a partition of $G$ into two disjoint induced subgraphs $C$ and $R$ such that $V(C)=V\left(C_{1}\right) \sqcup \cdots \sqcup V\left(C_{s}\right)$ where $C_{1}, \ldots, C_{s}$ are odd primitive cycles, and $R$ is bipartite. In this context, Theorem 6.5 gives necessary conditions for a graph to be a complete intersection by characterizing when $C$ is a complete intersection. Using this result, when $C$ is connected and $R$ is 2 -connected, Theorem 6.18 characterizes the complete intersection property on $G$ by determining all possible edges connecting $C$ and $R$.

In Section 2, we collect some results concerning general toric ideals that will be useful in the sequel. The main result in this section is Proposition 2.3, which deals with the problem of when the complete intersection property is preserved by elimination of variables. For toric ideals associated to graphs, Proposition 2.3 states that any induced subgraph of a complete intersection graph also has this property. This is Theorem 3.4 in Section 3, which allows 
us to obtain in Theorem 3.6 an upper bound for the number of edges of a complete intersection graph in terms of the number of vertices, improving all previously known bounds (see Corollary 3.7). An immediate consequence of Theorem 3.6 is that a complete intersection graph either has a vertex of degree $\leq 2$, or is 3-regular (see Corollary 3.8). Section 4 is devoted to designing Algorithm CI-graph, a polynomial time algorithm for checking whether a graph is a complete intersection. This algorithm is a direct consequence of Theorem 4.8 and works as follows: vertices of degree 1 are removed, also vertices of degree 2 are removed after checking certain conditions; if these conditions are not satisfied, the algorithm returns FALSE; otherwise, we iterate this process until we get either a trivial graph or a graph in which every vertex has degree $\geq 3$. If there is a vertex of degree $>3$, the algorithm returns False. Otherwise we use the characterization of complete intersection 3-regular graphs given in Theorem 4.4 . Finally, we use Theorem 2.4 to check if $G$ is a complete intersection. Section 5 deals with the problem of finding forbidden subgraphs in a complete intersection graph. The main result is Theorem 5.7, where we prove that odd theta graphs whose base vertices are not adjacent, and also even theta graphs, are forbidden subgraphs of a complete intersection graph (see Definition 5.4 for a definition of even and odd theta graphs). To prove this, we use Lemma[5.1 and Proposition 5.3, two technical results concerning the vertices of degree 2 in a complete intersection graph. In Section 6 we apply the previous results in order to obtain the above mentioned Theorem 6.5 and Theorem 6.18 together with their normal versions; Corollary 6.20 and Corollary 6.21

\section{Complete Intersection toric ideals}

In this section, $A$ denotes an $m \times n$ matrix with non-zero columns $a_{1}, \ldots, a_{n} \in \mathbb{N}^{m}$ and $P_{A} \subset k\left[x_{1}, \ldots, x_{n}\right]$ is the toric ideal of $A$, which is the kernel of the $k$-algebra homomorphism $\varphi: k\left[x_{1}, \ldots, x_{n}\right] \longrightarrow k\left[t_{1}, \ldots, t_{m}\right]$ induced by $\varphi\left(x_{i}\right)=t^{a_{i}}$

Definition 2.1. Let $T$ be a subset of $\left\{t_{1}, \ldots, t_{m}\right\}$. We define $T_{\varphi^{-1}}$ as the set $\left\{x_{i} \mid \varphi\left(x_{i}\right) \in k[T]\right\}$.

We have that $P_{A} \cap k\left[T_{\varphi^{-1}}\right]$ is the toric ideal associated to the matrix whose columns are the $i$-th columns of $A$ such that $x_{i} \in T_{\varphi^{-1}}$; see [27, Proposition 4.13(a)].

Lemma 2.2. Let $\mathfrak{B}$ be a set of generators of $P_{A}$ consisting of binomials, then $\mathfrak{B} \cap k\left[T_{\varphi^{-1}}\right]$ is a set of generators of $P_{A} \cap k\left[T_{\varphi^{-1}}\right]$. Moreover, if $\mathfrak{B}$ is minimal, then $\mathfrak{B} \cap k\left[T_{\varphi^{-1}}\right]$ is minimal.

Proof. Our proof begins the observation that whenever $f=x^{\alpha}-x^{\beta} \in P_{A}$, then $x^{\alpha} \in k\left[T_{\varphi^{-1}}\right]$ if and only if $x^{\beta} \in k\left[T_{\varphi^{-1}}\right]$, by the definition of $T_{\varphi^{-1}}$. Now let $g$ be a binomial in $P_{A} \cap k\left[T_{\varphi^{-1}}\right]$. Since $g \in P_{A}$, then $g=\sum_{f_{i} \in \mathfrak{B}} g_{i} f_{i}$ with $g_{i} \in$ $k\left[x_{1}, \ldots, x_{n}\right]$. Consider the morphism $\psi$ defined by $\psi\left(x_{i}\right)=x_{i}$ if $x_{i} \in T_{\varphi^{-1}}$, and $\psi\left(x_{i}\right)=0$ otherwise. Then for every $f_{i} \in \mathfrak{B}$, we get that $\psi\left(f_{i}\right)=f_{i}$ if $f_{i} \in k\left[T_{\varphi^{-1}}\right]$ or $\psi\left(f_{i}\right)=0$ otherwise. Thus,

$$
g=\psi(g)=\sum_{f_{i} \in \mathfrak{B}} \psi\left(g_{i}\right) \psi\left(f_{i}\right)=\sum_{f_{i} \in \mathfrak{B} \cap k\left[T_{\varphi^{-1}}\right]} \psi\left(g_{i}\right) f_{i} .
$$

Hence, $\mathfrak{B} \cap k\left[T_{\varphi^{-1}}\right]$ generates $P_{A} \cap k\left[T_{\varphi^{-1}}\right]$. Moreover, $\mathfrak{B} \cap k\left[T_{\varphi^{-1}}\right]$ is minimal whenever $\mathfrak{B}$ is.

Proposition 2.3. If $P_{A}$ is a complete intersection, then $P_{A} \cap k\left[T_{\varphi^{-1}}\right]$ is a complete intersection.

Proof. Let $\mathfrak{B}$ be a minimal set of generators of $P_{A}$ consisting of binomials, then $\mathfrak{B}$ is a regular sequence. Hence, by Lemma 2.2. the set $\mathfrak{B} \cap k\left[T_{\varphi^{-1}}\right]$ is a regular sequence which generates $P_{A} \cap k\left[T_{\varphi^{-1}}\right]$ and the result follows.

Note that Proposition 2.3 provides a new proof of [16, Theorem 4.1] for the particular case of a toric ideal. 
In the following sections we will use Theorem 1.1 in [15], which is a reformulation of [8, Theorem 2.9]. For presenting this result we have to introduce first some definitions.

Let $B$ be an integral matrix, $B$ is called mixed if every row of $B$ has a positive and a negative entry. $B$ is said to be dominating if it does not contain any square mixed submatrix. $\Delta_{t}(B)$ denotes the greatest common divisor of every $t \times t$ minor of $B$ where $t \leq \operatorname{rank}(B)$.

Theorem 2.4. ([15, Theorem 1.1], [8, Theorem 2.9]) Let $P_{A}$ be a toric ideal of height $r$ and $g_{i}=x^{\alpha_{i}}-x^{\beta_{i}} \in P_{A}$ with $\operatorname{gcd}\left(x^{\alpha_{i}}, x^{\beta_{i}}\right)=1$ for $1 \leq i \leq r$. If $B$ denotes the $r \times n$ matrix whose $i$-th row is $\alpha_{i}-\beta_{i}$ for $1 \leq i \leq r$, then

$$
P_{A}=\left(g_{1}, \ldots, g_{r}\right) \Longleftrightarrow B \text { is dominating and } \Delta_{r}(B)=1 \text {. }
$$

The following result, whose proof is straightforward, will be useful to prove that certain matrices are dominating.

Lemma 2.5. Let $B$ be an $r \times n$ matrix with column vectors $c_{1}, \ldots, c_{n} \in \mathbb{Z}^{r}$ such that $c_{i}$ has only one nonzero entry for some $1 \leq i \leq n$, and denote by $B^{\prime}$ the $r \times n-1$ matrix with column vectors $c_{1}, \ldots, c_{i-1}, c_{i+1}, \ldots, c_{n}$. Then, $B$ is dominating if and only if $B^{\prime}$ is dominating.

\section{An upper bound for the number of edges in a complete intersection graph}

We begin this section by setting up some notation and terminology about graphs. For unexplained terminology and results on graphs we refer to [4, 13].

A walk $w$ connecting $u, v \in V(G)$ is a finite sequence of vertices $w=\left(u=v_{i_{0}}, v_{i_{1}}, \ldots, v_{i_{q}}=v\right)$ such that $\left\{v_{i_{j-1}}, v_{i_{j}}\right\} \in$ $E(G)$ for every $1 \leq j \leq q$. If $v_{i_{j}} \neq v_{i_{k}}$ for every $0 \leq j<k \leq q$ then $w$ is called a path. The vertex set of the walk $w$ is $V(w):=\left\{v_{i_{0}}, \ldots, v_{i_{q}}\right\}$ and its edge set is $E(w):=\left\{\left\{v_{i_{j-1}}, v_{i_{j}}\right\} \mid 1 \leq j \leq q\right\}$. The length of the walk is the number $q$ of edges in the walk. An even (respectively odd) walk is a walk of even (respectively odd) length. A walk is closed if $u=v$. A cycle is a closed walk with $v_{i_{k}} \neq v_{i_{j}}$ for every $1 \leq k<j \leq q$. A cycle is primitive if $\left\{v_{i_{k}}, v_{i_{j}}\right\} \notin E(G)$ for every $1 \leq k<k+1<j \leq q$.

For a walk $w=\left(u=v_{i_{0}}, v_{i_{1}}, \ldots, v_{i_{q}}=v\right)$ we denote by $-w$ the inverse walk $\left(v=v_{i_{q}}, \ldots, v_{i_{1}}, v_{i_{0}}=u\right)$. Let $w_{1}, \ldots, w_{r}$ be walks such that $w_{i}$ connects $u_{i}, u_{i+1}$ for every $i \in\{1, \ldots, r\}$, then $\left(w_{1}, \ldots, w_{r}\right)$ denotes the walk connecting $u_{1}, u_{r+1}$ obtained by sticking the walks $w_{1}, \ldots, w_{r-1}$ and $w_{r}$ together.

Given an even closed walk, $w=\left(v_{i_{0}}, \ldots, v_{i_{2 q}}=v_{i_{0}}\right)$ where $e_{k_{j}}=\left\{v_{i_{j-1}}, v_{i_{j}}\right\}$ for $1 \leq j \leq 2 q$, we denote by $B_{w}$ the binomial

$$
B_{w}:=\prod_{l=1}^{q} x_{k_{2 l-1}}-\prod_{l=1}^{q} x_{k_{2 l}} .
$$

Villarreal [29, Proposition 3.1] proved that $P_{G}$ is generated by these binomials, i.e., $P_{G}=\left(\left\{B_{w} \mid w\right.\right.$ is an even closed walk\}). Hibi and Ohsugi [21, Lemma 3.2] improved this result by giving a necessary condition for a binomial in $P_{G}$ to be primitive. Recall that $x^{\alpha}-x^{\beta} \in P_{G}$ is primitive if there exists no other binomial $x^{\alpha^{\prime}}-x^{\beta^{\prime}} \in P_{G}$ such that $x^{\alpha^{\prime}} \mid x^{\alpha}$ and $x^{\beta^{\prime}} \mid x^{\beta}$. Whenever a binomial belongs to a minimal set of generators of $P_{G}$, then it is necessarily primitive (see [27]); thus the set of all primitive binomials of $P_{G}$, which is called the Graver basis of $P_{G}$, is a set of generators for $P_{G}$.

Lemma 3.1. [21, Lemma 3.2] If $B_{w}$ is primitive, then one of these holds:

- $w$ is an even cycle, 
- $w=\left(C_{1}, C_{2}\right)$ where $C_{1}$ and $C_{2}$ are odd cycles having exactly a vertex in common, or

- $w=\left(C_{1}, w_{1}, C_{2},-w_{2}\right)$ where $C_{1}, C_{2}$ are vertex disjoint odd cycles and $w_{1}, w_{2}$ are walks connecting a vertex $v_{1} \in V\left(C_{1}\right)$ and a vertex $v_{2} \in V\left(C_{2}\right)$.

For a complete characterization of primitive binomials and a description of all minimal sets of generators of $P_{G}$ formed by binomials we refer the reader to [23].

Now we aim to prove that the complete intersection is hereditary, i.e., if a graph is a complete intersection then every induced subgraph also is. Let us first recall the definition of induced subgraph.

Definition 3.2. Let $G$ be a graph, $G^{\prime}$ is an induced subgraph of $G$ if $V\left(G^{\prime}\right) \subset V(G)$ and

$$
E\left(G^{\prime}\right)=\left\{e \in E(G) \mid e \subset V\left(G^{\prime}\right)\right\}
$$

If $V^{\prime} \subset V(G)$, we will denote by $\left[V^{\prime}\right]$ the induced subgraph of $G$ with vertex set $V^{\prime}$. Let $v_{1}, \ldots, v_{s}$ be vertices of $G$, the induced subgraph $\left[V(G) \backslash\left\{v_{1}, \ldots, v_{s}\right\}\right]$ will also be denoted by $G \backslash\left\{v_{1}, \ldots, v_{s}\right\}$.

For an induced subgraph $G^{\prime}$, if we denote $T:=\left\{t_{i} \mid v_{i} \in V\left(G^{\prime}\right)\right\}$, then $T_{\varphi^{-1}}=\left\{x_{i} \mid e_{i} \in E\left(G^{\prime}\right)\right\}$ and $P_{G^{\prime}}=P_{G} \cap k\left[T_{\varphi^{-1}}\right]$. Hence, by Lemma 2.2 and Proposition 2.3 we deduce the following results.

Proposition 3.3. Let $G^{\prime}$ be an induced subgraph of $G$. If $P_{G}=\left(B_{w_{1}}, \ldots, B_{w_{s}}\right)$ for some even closed walks $w_{1}, \ldots, w_{s}$ in $G$, then $P_{G^{\prime}}=\left(B_{w_{i}} \mid V\left(w_{i}\right) \subset V\left(G^{\prime}\right), 1 \leq i \leq s\right)$.

Theorem 3.4. Let $G^{\prime}$ be an induced subgraph of $G$. If $G$ is a complete intersection, then so is $G^{\prime}$.

A different proof of Theorem 3.4 exists also in [28, Theorem 3.1].

These results are not true in general if we drop the assumption that $G^{\prime}$ is induced, as the example in Figure 3 shows.
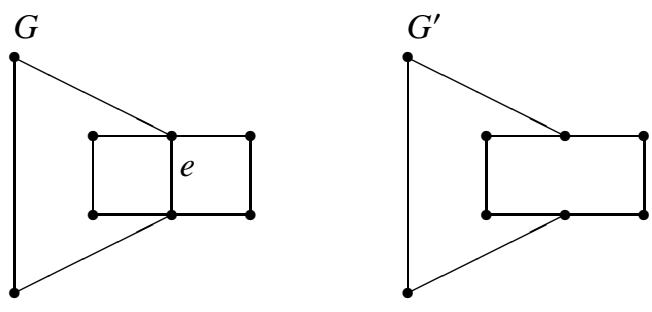

Figure 3: $G^{\prime}$ is a subgraph of $G$, both are bipartite but $G$ is a ring graph and $G^{\prime}$ is not. Thus $G$ is a complete intersection and $G^{\prime}$ is not.

An almost immediate consequence of Theorem 3.4 is that a graph is a complete intersection if and only if all its connected components are complete intersections. This allows us to reduce our study to connected graphs.

Corollary 3.5. Let $G$ be a graph with connected components $G_{1}, \ldots, G_{s}$. Then, $G$ is a complete intersection if and only if so are $G_{1}, \ldots, G_{s}$.

Proof. One implication follows by Theorem 3.4 because $G_{i}$ is an induced subgraph of $G$ for $1 \leq i \leq s$. Assume now that $G_{1}, \ldots, G_{s}$ are complete intersections and let $\mathfrak{B}_{i}$ be a minimal set of generators of $P_{G_{i}}$ for $1 \leq i \leq s$. Since 
$P_{G}=\left(\left\{B_{w} \mid w\right.\right.$ is an even closed walk $\left.\}\right)$ and every even closed walk is necessarily contained in a connected component of $G$, it is evident that $P_{G}=\left\langle\mathfrak{B}_{1} \cup \cdots \cup \mathfrak{B}_{s}\right\rangle$. Moreover, we have that $\operatorname{ht}\left(P_{G}\right)=\operatorname{ht}\left(P_{G_{1}}\right)+\cdots+\operatorname{ht}\left(P_{G_{s}}\right)$, and the result follows.

For a vertex $v \in V(G)$, the neighborhood of $v$ is the set of vertices which are adjacent to $v$, i.e., $N_{G}(v):=\{u \in$ $V(G) \mid\{u, v\} \in E(G)\}$. The cardinality of this set is called the degree of $v$ and is denoted by $\operatorname{deg}_{G}(v), \operatorname{or} \operatorname{deg}(v)$ when $G$ is understood. $G$ is $k$-regular if every vertex of $G$ has degree $k$.

The following result provides an upper bound for the number of edges of complete intersection graphs. This gives the taste that they can not be very dense.

Theorem 3.6. Let $G$ be a complete intersection connected graph, then:

- $2|E(G)|+4 \leq 4|V(G)|-\sum_{v \in V(G)} b(G \backslash\{v\})$ if $G$ is bipartite.

- $2|E(G)| \leq 3|V(G)|-\sum_{v \in V(G)} b(G \backslash\{v\})$ if $G$ is not bipartite.

In both cases equality is attained if and only if $P_{G}$ is generated by quadrics.

Proof. Let $\left\{B_{w_{1}}, \ldots, B_{w_{r}}\right\}$ be a minimal set of generators of $P_{G}$, where $w_{i}$ is an even closed walk for every $1 \leq i \leq r$. By Proposition 3.3. for every $v \in V(G)$ we have that $P_{G \backslash\{v\}}=\left(B_{w_{i}} \mid v \notin V\left(w_{i}\right), 1 \leq i \leq r\right)$ and by Lemma 3.1, it follows that $\left|V\left(w_{i}\right)\right| \geq 4$ and $\left|V\left(w_{i}\right)\right|=4$ if and only if $w_{i}$ is a cycle of length 4 , which is equivalent to $B_{w_{i}}$ is a quadric.

Therefore,

$$
4 \mu\left(P_{G}\right) \leq \sum_{v \in V(G)} \mu\left(P_{G}\right)-\mu\left(P_{G \backslash\{v\}}\right)
$$

and equality holds if and only if $P_{G}$ is generated by quadrics. Now suppose that $G$ is a complete intersection then, by Theorem 3.4, $G \backslash\{v\}$ is a complete intersection for every $v \in V(G)$. Hence,

$$
\begin{gathered}
4 \operatorname{ht}\left(P_{G}\right) \leq \sum_{v \in V(G)}\left(\mu\left(P_{G}\right)-\mu\left(P_{G \backslash\{v\}}\right)\right)=\sum_{v \in V(G)}\left(\operatorname{ht}\left(P_{G}\right)-\operatorname{ht}\left(P_{G \backslash\{v\}}\right)\right)= \\
=\sum_{v \in V(G)}(\operatorname{deg}(v)-1+b(G)-b(G \backslash\{v\}))=2 n-m+b(G) m-\sum_{v \in V(G)} b(G \backslash\{v\}) .
\end{gathered}
$$

If $G$ is bipartite, then $b(G)=1, \operatorname{ht}\left(P_{G}\right)=n-m+1$ and $2 n+4 \leq 4 m-\sum_{v \in V(G)} b(G \backslash\{v\})$, and if $G$ is not bipartite, then $b(G)=0, \operatorname{ht}\left(P_{G}\right)=n-m$ and $2 n \leq 3 m-\sum_{v \in V(G)} b(G \backslash\{v\})$. In both cases equality is attained if and only if $P_{G}$ is generated by quadrics.

Katzman in [17, Corollary 3.8] proved that $|E(G)|+4 \leq 2|V(G)|$ for a complete intersection connected bipartite graph. Independently, from a result of Fischer, Morris and Shapiro [6, Corollary 3.4] one can deduce that if $G$ is a complete intersection connected graph, then $|E(G)|+4 \leq 2|V(G)|$ if $G$ is bipartite and $|E(G)|+2 \leq 2|V(G)|$ if $G$ is non bipartite. The following result improves these bounds.

Corollary 3.7. Let $G$ be a complete intersection connected graph, then

- $2|E(G)|+4 \leq 3|V(G)|$ if $G$ is bipartite, and

- $2|E(G)| \leq 3|V(G)|$ if $G$ is not bipartite.

Proof. It is a consequence of Theorem 3.6 and that if $G$ is bipartite, then $b(G \backslash\{v\}) \geq 1$ for every $v \in V(G)$.

This section ends with two more consequences of Theorem 3.6 . 
Corollary 3.8. Let $G$ be a complete intersection graph, then either

(a) there exists a vertex of degree $\leq 2$, or

(b) $G$ is 3-regular, $b(G \backslash\{v\})=0$ for every $v \in V(G)$ and $P_{G}$ is generated by quadrics.

Proof. Assume that $G$ is a connected graph and every vertex has degree $\geq 3$. Then $2 n=\sum_{v \in V(G)} \operatorname{deg}(v) \geq 3 m$; hence by Corollary 3.7 $G$ is not bipartite and $2 n=3 m$. Thus, $G$ is 3-regular and by Theorem 3.6 this can only happen if (b) holds.

We denote by $\mathcal{K}_{m}$ the complete graph with $m$ vertices and by $\mathcal{K}_{m_{1}, m_{2}}$ the complete bipartite graph with partitions of sizes $m_{1}$ and $m_{2}$.

Corollary 3.9. If $G$ is a complete intersection, then it does not contain $\mathcal{K}_{2,3}$ as a subgraph.

Proof. Assume that $G$ contains $\mathcal{K}_{2,3}$ as a subgraph and denote by $H$ the induced subgraph of $G$ with 5 vertices containing $\mathcal{K}_{2,3}$ as a subgraph. If $H=\mathcal{K}_{2,3}$ then $2|E(H)|+4=16>15=3|V(H)|$. If $E(H)=E\left(\mathcal{K}_{2,3}\right) \cup\left\{e_{1}, \ldots, e_{s}\right\}$, we have that $H$ is not bipartite and if $s=1$ and $e_{1}=\left\{v_{1}, v_{2}\right\} \subset V(H)$, then $b\left(H \backslash\left\{v_{i}\right\}\right) \geq 1$ for $i=1,2$. So

$$
2|E(H)|=12+2 s>15+2(s-2) \geq 3|V(H)|-\sum_{v \in V(H)} b(H \backslash\{v\}) .
$$

In both cases, one gets that $H$ is not a complete intersection by Theorem 3.6 and Corollary 3.7 Furthermore, by Theorem 3.4 one concludes that $G$ is not a complete intersection.

\section{The algorithm}

The aim of this section is to provide Algorithm CI-graph, an algorithm for checking whether a graph is a complete intersection. This algorithm follows as a consequence of Theorem 4.8, which is the main result of this section.

By Corollary 3.8 we have that a complete intersection graph either has a vertex of degree $\leq 2$ or is 3 -regular. This section begins with a thorough study of 3-regular complete intersection graphs. It will turn out in Theorem 4.4 that a 3 -regular graph is a complete intersection if and only if it is an odd band or an even Möbius band. To prove this we need some definitions and a technical lemma. Theorem 4.4 will be essential for proving Theorem 4.8

Definition 4.1. A chain is a graph $G$ with $V(G)=\left\{a_{1}, \ldots, a_{r}, b_{1}, \ldots, b_{r}\right\}$ and edges $\left\{a_{i}, a_{i+1}\right\},\left\{b_{i}, b_{i+1}\right\}$ and $\left\{a_{j}, b_{j}\right\}$ for $1 \leq i<r, 1 \leq j \leq r$.

Definition 4.2. Let $G$ be a graph with a subgraph $H$ such that $V(G)=V(H)$ and $H$ is a chain. If $E(G)=E(H) \cup$ $\left\{\left\{a_{1}, a_{r}\right\},\left\{b_{1}, b_{r}\right\}\right\}$ we say that $G$ is a band. If $E(G)=E(H) \cup\left\{\left\{a_{1}, b_{r}\right\},\left\{a_{r}, b_{1}\right\}\right\}$ we say that $G$ is a Möbius-band. In addition if $r$ is odd, we say that $G$ is an odd Möbius-band (or odd band) and if $r$ is even, we say that $G$ is an even Möbius-band (or even band).

Lemma 4.3. Let $G$ be a complete intersection connected 3-regular graph. Then, either $G=\mathcal{K}_{4}$ or for every $v \in V(G)$ there exists a chain subgraph $H$ of $G$ with 6 vertices, such that $v \in V(H)$ and $\operatorname{deg}_{H}(v)=3$. 
Proof. Firstly note that $P_{G}$ is generated by quadrics and $b(G \backslash\{v\})=0$ for every $v \in V(G)$ by Corollary 3.8, in particular, $G$ is not bipartite. Let $\mathfrak{B}:=\left\{B_{w_{1}}, \ldots, B_{w_{r}}\right\}$ be a minimal set of generators of $P_{G}$ where $w_{i}$ is a length four cycle for $1 \leq i \leq r$. Take $v \in V(G)$ and denote by $u_{1}, u_{2}, u_{3}$ its neighbors. From one hand, we have that

$$
\operatorname{ht}\left(P_{G}\right)-\operatorname{ht}\left(P_{G \backslash\{v\}}\right)=\operatorname{deg}(v)-1+b(G)-b(G \backslash\{v\})=2,
$$

and by Proposition 3.3 and Theorem 3.4, $P_{G \backslash\{v\}}$ is a complete intersection minimally generated by $\left\{B_{w_{i}} \mid v \notin V\left(w_{i}\right)\right\}$; thus $\left|\left\{w_{i} \mid v \in V\left(w_{i}\right), 1 \leq i \leq r\right\}\right|=2$, and we can assume that $v \in V\left(w_{1}\right) \cap V\left(w_{2}\right)$. From the other hand, $\mid N_{G}\left(u_{i}\right) \cap$ $N_{G}\left(u_{j}\right) \mid \leq 2$ for every $1 \leq i<j \leq 3$; otherwise $\mathcal{K}_{2,3}$ is a subgraph of $G$, which is impossible by Corollary 3.9 . Thus we can assume that $w_{1}=\left(v, u_{1}, v_{1}, u_{2}, v\right)$ and $w_{2}=\left(v, u_{2}, v_{2}, u_{3}, v\right)$ for some $v_{1}, v_{2} \in V(G)$. Since $w_{1}$ and $w_{2}$ are length 4 cycles and $\mathcal{K}_{2,3}$ is not a subgraph of $G$, we see that $v_{1} \neq v_{2}$.

If $v_{1}=u_{3}$ or $v_{2}=u_{1}$, then $G=\mathcal{K}_{4}$. Otherwise there is a chain subgraph $H$ of $G$ with $V(H)=\left\{v, u_{1}, u_{2}, u_{3}, v_{1}, v_{2}\right\}$ and $\operatorname{deg}_{H}(v)=3$.

Theorem 4.4. Let $G$ be a 3-regular graph. Then, $G$ is a complete intersection if and only if the connected components of $G$ are odd bands or even Möbius bands.

Proof. By Corollary 3.5 we can assume that $G$ is connected.

$(\Rightarrow)$ Since $\mathcal{K}_{4}$ is an even Möbius band we will assume $G \neq \mathcal{K}_{4}$. By Corollary 3.8 we get that $G$ is a 3-regular graph with $b(G \backslash\{v\})=0$ for every $v \in V(G)$ and by Lemma 4.3 there exists a subgraph $H^{\prime}$ of $G$ which is a chain with 6 vertices. Choose $H$ a chain subgraph of $G$ maximal with respect to $|V(H)|$; then $V(H)=\left\{a_{1}, \ldots, a_{r}, b_{1}, \ldots, b_{r}\right\}$ for some $r \geq 3$. Applying Lemma 4.3 with $v=a_{r}$, we get that there exist $a_{r+1}, b_{r+1} \in V(G)$ such that $\left\{a_{r}, a_{r+1}\right\},\left\{b_{r}, b_{r+1}\right\}$ and $\left\{a_{r+1}, b_{r+1}\right\} \in E(G)$. We will prove that $\left\{a_{1}, b_{1}\right\}=\left\{a_{r+1}, b_{r+1}\right\}$.

By the maximality of $H$ either $a_{r+1}$ or $b_{r+1}$ belong to $V(H)$. We assume that $a_{r+1} \in V(H)$, then $a_{r+1}=a_{1}$ or $a_{r+1}=b_{1}$. If $a_{r+1}=a_{1}$, by Lemma 4.3. one can conclude that $b_{r+1}=b_{1}$ because $\left\{a_{1}, b_{r+1}\right\},\left\{b_{r}, b_{r+1}\right\} \in E(G)$ and $a_{2} \neq a_{r}$. If $a_{r+1}=b_{1}$, since $\left\{b_{1}, b_{r+1}\right\},\left\{b_{r}, b_{r+1}\right\} \in E(G)$, one can conclude that either $b_{r+1}=a_{1}$ or $b_{r+1}=b_{2}$ and $r=3$. Furthermore, if $b_{r+1}=b_{2}$ and $r=3$ then there is a $\mathcal{K}_{2,3}$ subgraph with vertices $a_{1}, a_{2}, a_{3}, b_{1}, b_{2}$ and this is not possible by Corollary 3.9

Therefore we have proved that $H$ is either a band or a Möbius band and $G$ is 3-regular and connected, then $G=H$. Finally, $G$ can be neither an even band nor an odd Möbius band, because both are bipartite and by Corollary $3.7 G$ is not bipartite.

$(\Leftarrow)$ Denote $e_{i}:=\left\{a_{i}, b_{i}\right\}$ for $1 \leq i \leq r, e_{r+i}:=\left\{a_{i}, a_{i+1}\right\}$ and $e_{2 r+i}:=\left\{b_{i}, b_{i+1}\right\}$ for $1 \leq i<r$. If $G$ is an odd band, we set $e_{2 r}:=\left\{a_{1}, a_{r}\right\}$ and $e_{3 r}:=\left\{b_{1}, b_{r}\right\}$ and if $G$ is an even Möbius band, we set $e_{2 r}:=\left\{a_{1}, b_{r}\right\}$ and $e_{3 r}:=\left\{a_{r}, b_{1}\right\}$. In both cases $G$ is not bipartite, furthermore $G$ has $3 r$ edges and $2 r$ vertices, then $\operatorname{ht}\left(P_{G}\right)=r$.

Let $w_{i}$ be the length 4 cycle $w_{i}:=\left(a_{i}, b_{i}, b_{i+1}, a_{i+1}, a_{i}\right)$ for $1 \leq i \leq r-1$. If $G$ is an odd band, we denote $w_{r}:=\left(a_{1}, b_{1}, b_{r}, a_{r}, a_{1}\right)$ and if $G$ is an even Möbius band we denote $w_{r}:=\left(a_{1}, b_{1}, a_{r}, b_{r}, a_{1}\right)$. In both cases we have that $B_{w_{i}}=x_{i} x_{i+1}-x_{r+i} x_{2 r+i}$ for $1 \leq i<r$ and $B_{w_{r}}=x_{1} x_{r}-x_{2 r} x_{3 r}$. We denote by $\left\{e_{1}, \ldots, e_{3 r}\right\}$ the canonical basis of $\mathbb{Z}^{r}$, $\gamma_{i}:=e_{i}+e_{i+1}-e_{r+i}-e_{2 r+i}$ for $1 \leq i<r, \gamma_{r}=e_{1}+e_{r}-e_{2 r}-e_{3 r}$ and $B$ the $r \times 3 r$ matrix whose rows are $\gamma_{1}, \ldots, \gamma_{r}$. Then $\Delta_{r}(B)=1$. Let $B^{\prime}$ be the $r \times r$ submatrix of $B$ consisting of its first $r$ columns. Since every entry of $B^{\prime}$ is nonnegative we get that $B^{\prime}$ is dominating. Furthermore, for every $j>r$ the $j$-th column of $B$ has only one nonzero entry whose value is -1 , then by Lemma $2.5 \mathrm{~B}$ is dominating. By Theorem 2.4 we can conclude that $G$ is a complete intersection.

The proof above gives more, whenever $G$ is an odd band or an even Möbius band we have obtained a minimal set of generators of the ideal. 

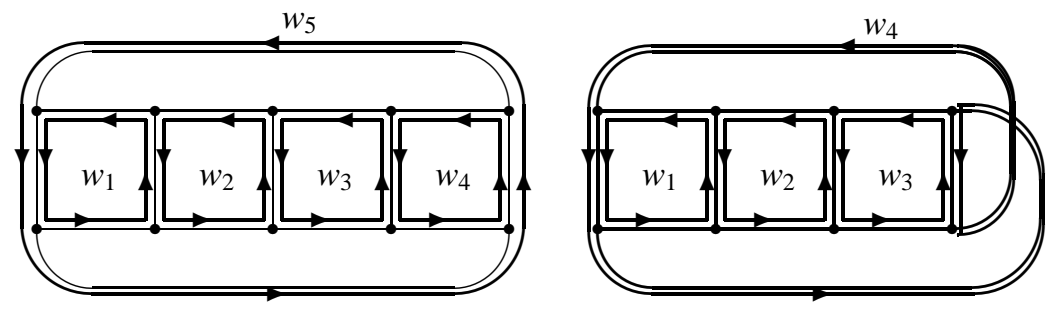

Figure 4: An odd band, an even Möbius band and the even closed walks corresponding to a minimal set of generators of each.

Corollary 4.5. Let $G$ be an odd band, then $P_{G}=\left(B_{w_{1}}, \ldots, B_{w_{r}}\right)$, where $w_{i}:=\left(a_{i}, b_{i}, b_{i+1}, a_{i+1}, a_{i}\right)$ for $1 \leq i \leq r-1$ and $w_{r}:=\left(a_{1}, b_{1}, b_{r}, a_{r}, a_{1}\right)$.

Corollary 4.6. Let $G$ be an even Möbius band, then $P_{G}=\left(B_{w_{1}}, \ldots, B_{w_{r}}\right)$, where $w_{i}:=\left(a_{i}, b_{i}, b_{i+1}, a_{i+1}, a_{i}\right)$ for $1 \leq i \leq$ $r-1$ and $w_{r}:=\left(a_{1}, b_{1}, a_{r}, b_{r}, a_{1}\right)$.

Remark 4.7. Since every even Möbius band except $\mathcal{K}_{4}$ is not planar, Theorem 4.4 provides an infinite family of non planar complete intersection graphs. Both Katzman [17] and Gitler, Reyes and Villarreal [11] proved that whenever $G$ is a bipartite complete intersection then it is planar. As one can see this result is no longer true if we drop the assumption that $G$ is bipartite. This was first realized by Katzman [17, Remark 3.9], who provided a Möbius band with 8 vertices as an example of a complete intersection non planar graph. Later Tatakis and Thoma [28] provided another example which is not a Möbius band.

We are thus led to the main result of this section.

Theorem 4.8. Let $G$ be a graph without isolated vertices. Then, $G$ is a complete intersection if and only if one of the following holds

1. $\exists v \in V(G)$ of degree 1 and $G \backslash\{v\}$ is a complete intersection.

2. $\exists v \in V(G)$ of degree 2 such that $b(G \backslash\{v\})=b(G)+1$ and $G \backslash\{v\}$ is a complete intersection.

3. $\exists v \in V(G)$ of degree 2 such that $b(G \backslash\{v\})=b(G), G \backslash\{v\}$ is a complete intersection and exists a shortest even closed walk $w$ with

$$
V(w)=\{v\} \cup N_{G}(v) \cup\{u \in V(G) \mid b(G \backslash\{u, v\})>b(G \backslash\{u\})\},
$$

such that

$$
P_{G}=P_{G \backslash\{v\}} \cdot k\left[x_{1}, \ldots, x_{n}\right]+\left(B_{w}\right) .
$$

4. The connected components of $G$ are odd bands or even Möbius bands.

Proof. Our proof starts with the observation that if $v \in V(G)$ has degree 1 , then $b(G \backslash\{v\})=b(G)$ and if it has degree 2 , then $b(G \backslash\{v\})-b(G) \in\{0,1\}$. Thus, the proof falls naturally in the following four cases:

(a) there exists $v \in V(G)$ such that $\operatorname{deg}(v)=1$,

(b) there exists $v \in V(G)$ such that $\operatorname{deg}(v)=2$ and $b(G \backslash\{v\})=b(G)+1$,

(c) there exists $v \in V(G)$ such that $\operatorname{deg}(v)=2$ and $b(G \backslash\{v\})=b(G)$ or

(d) $\operatorname{deg}(v)>2$ for every $v \in V(G)$.

We observe that $J:=P_{G \backslash\{v\}} \cdot k\left[x_{1}, \ldots, x_{n}\right]$ is a prime ideal and $J \subset P_{G}$. If (a) or (b) holds, then ht $\left(P_{G \backslash\{v\}}\right)=\operatorname{ht}\left(P_{G}\right)$, this yields $P_{G}=J$ and $G$ is a complete intersection if and only if $G \backslash\{v\}$ is a complete intersection. 
If (c) holds, then $\operatorname{ht}\left(P_{G}\right)=\operatorname{ht}\left(P_{G \backslash\{v\}}\right)+1$. If $G \backslash\{v\}$ is a complete intersection and there exists an even closed walk $w$ in $G$ such that $P_{G}=J+\left(B_{w}\right)$, then $G$ is evidently a complete intersection. Suppose that $G$ is a complete intersection and let $w_{1}, \ldots, w_{r}$ be even closed walks in $G$ such that $P_{G}=\left(B_{w_{1}}, \ldots, B_{w_{r}}\right)$ with $r=\operatorname{ht}\left(P_{G}\right)$. By Proposition 3.3 and Theorem 3.4 we have that $P_{G \backslash\{v\}}$ is a complete intersection minimally generated by $\left\{B_{w_{i}} \mid v \notin V\left(w_{i}\right)\right\}$. Since $\operatorname{ht}\left(P_{G \backslash\{v\}}\right)=r-1$, there exists a unique $i \in\{1, \ldots, r\}$ such that $v \in V\left(w_{i}\right)$ and $P_{G}=J+\left(B_{w_{i}}\right)$. It is obvious that $N_{G}(v):=\left\{v_{1}, v_{2}\right\} \subset V\left(w_{i}\right)$ because $v \in V\left(w_{i}\right), \operatorname{deg}(v)=2$ and $B_{w_{i}}$ is primitive. Now, again by Proposition 3.3 and Theorem 3.4 for every $u \in V(G) \backslash\left\{v, v_{1}, v_{2}\right\}$, we have that $u \in V\left(w_{i}\right)$ if and only if $\left\{B_{w_{j}} \mid u \notin V\left(w_{j}\right)\right\}=\left\{B_{w_{j}} \mid v, u \notin\right.$ $\left.V\left(w_{j}\right)\right\}$, or equivalently if $\mu\left(P_{G \backslash\{u\}}\right)=\mu\left(P_{G \backslash\{u, v\}}\right) \Leftrightarrow \operatorname{ht}\left(P_{G \backslash\{u\}}\right)=\operatorname{ht}\left(P_{G \backslash\{u, v\}}\right)$. Since $\operatorname{deg}_{G \backslash\{u\}}(v)=2$, this is equivalent to $b(G \backslash\{u, v\})>b(G \backslash\{u\})$.

Finally, if (d) holds, Corollary 3.8 and Theorem 4.4 complete the proof.

This theorem yields Algorithm CI-graph, see Figure 5, an algorithm to determine if a graph is a complete intersection. This method begins by removing all the vertices of degree 1 and 2 iteratively. Whenever we remove a vertex $v$ of degree 2, we check whether $b(G)=b(G \backslash\{v\})$. In the positive case, we construct a set $W \subset V(G)$ and look for an even closed walk $w$ such that $V(w)=W$. If such a walk does not exist, then $G$ is not a complete intersection, otherwise we take $w$ a shortest even walk such that $V(w)=W$ and define the binomial $B_{w}$; one can obtain such an even walk in polynomial time by means of the algorithm proposed in [18]. Once we have removed every vertex of degree $\leq 2$, either we get a trivial graph or we reach a graph $G^{\prime}$ where every vertex has degree $>2$. If there exists a connected component of $G^{\prime}$ which is neither an odd band nor an even Möbius band, then $G$ is not a complete intersection. Otherwise we can construct a set of $r=\operatorname{ht}\left(P_{G}\right)$ binomials $\left\{B_{w_{1}}, \ldots, B_{w_{r}}\right\} \subset P_{G}$, and $G$ is a complete intersection if and only if $P_{G}=\left(B_{w_{1}}, \ldots, B_{w_{r}}\right)$. For checking this equality we use Theorem 2.4. It is worth pointing out that in [7] the authors give a polynomial algorithm to decide if a matrix is dominating; thus one can check if the equality $P_{G}=\left(B_{w_{1}}, \ldots, B_{w_{r}}\right)$ holds in polynomial time.

As a direct consequence of this algorithm we have the following result.

Corollary 4.9. The problem of determining whether a graph is a complete intersection is in the complexity class $\mathcal{P}$.

Proof. Counting the number of connected components of a graph and deciding whether a graph is bipartite, and thus computing $b(H)$, can be done in polynomial time for every graph $H$. To prove the result it only remains to prove that, given a connected graph $H$, one can decide if $H$ is either an odd band or an even Möbius band in polynomial time. For this purpose we propose a polynomial time algorithm that returns True if $H$ is an odd band or an even Möbius band, or FALSE otherwise. If $H$ has 4 vertices then we return TRUE if and only if $H=\mathcal{K}_{4}$. If $H$ has more than 4 vertices, the algorithm lies on the fact if $H$ is an odd band or an even Möbius band with $V(H)=\left\{a_{1}, \ldots, a_{r}, b_{1}, \ldots, b_{r}\right\}$ and edges $\left\{a_{i}, a_{i+1}\right\},\left\{b_{i}, b_{i+1}\right\}$ for all $i \in\{1, \ldots, r-1\},\left\{a_{i}, b_{i}\right\}$ for all $i \in\{1, \ldots, r\}$; then $b\left(H \backslash\left\{a_{i}, b_{i}\right\}\right)=1$ for all $i \in\{1, \ldots, r\}, b\left(H \backslash\left\{a_{i}, a_{i+1}\right\}\right)=0$ for every $i \in\{1, \ldots, r-1\}$; moreover $b\left(H \backslash\left\{a_{1}, a_{r}\right\}\right)=0$ if $H$ is an odd band, and $b\left(H \backslash\left\{a_{1}, b_{r}\right\}\right)=0$ if $H$ is an even Möbius band. The algorithm receives as input the graph $H$, if $H$ is not 3-regular or $H$ is bipartite, we return FALSE. Otherwise we take $a_{1} \in V(H)$ an arbitrary vertex and denote $N_{H}\left(a_{1}\right):=\left\{w_{1}, w_{2}, w_{3}\right\}$. We compute $c_{i}:=b\left(H \backslash\left\{a_{1}, w_{i}\right\}\right)$ for all $i \in\{1,2,3\}$ and assume that $c_{1} \geq c_{2} \geq c_{3}$. If $c_{2} \geq 1$ or $c_{1}=0$, then we return FALSE. Otherwise we set $b_{1}:=w_{1}$. Now we take $a_{2} \in N_{H}\left(a_{1}\right)$, such that $a_{2} \notin H_{1}:=\left\{a_{1}, b_{1}\right\}$ and iterate this process until we get that $V(H)=\left\{a_{1}, \ldots, a_{r}, b_{1}, \ldots, b_{r}\right\},\left\{a_{i}, a_{i+1}\right\} \in E(H)$ for all $i \in\{1, \ldots, r-1\}$ and $\left\{a_{i}, b_{i}\right\} \in E(H)$ for all $i \in\{1, \ldots, r\}$. Thus, we return TRUE if and only if $\left\{b_{i}, b_{i+1}\right\} \in E(H)$ for all $i \in\{1, \ldots, r-1\}$.

Let us illustrate how Algorithm CI-graph works with an example.

Example 4.10. Let us prove that the graph $G$ in Figure 6 is not a complete intersection.

Firstly, we observe that $\operatorname{deg}_{G}\left(v_{7}\right)=2$ and $b(G)=0=b\left(G \backslash\left\{v_{7}\right\}\right)$. We set $W_{1}:=\left\{v_{7}\right\} \cup N_{G}\left(v_{7}\right) \cup\{u \in V(G) \mid b(G \backslash$ $\left.\left.\left\{u, v_{7}\right\}\right)>b(G \backslash\{u\})\right\}=\left\{v_{3}, v_{4}, v_{6}, v_{7}\right\}$. We construct $w_{1}$ a shortest even closed walk with $V\left(w_{1}\right)=W_{1}$. Thus $w_{1}$ is the length four cycle $w_{1}=\left(v_{7}, v_{3}, v_{4}, v_{6}, v_{7}\right)$ and $B_{w_{1}}=x_{4} x_{8}-x_{7} x_{9}$. 


\section{Algorithm CI-graph}

Input: $\quad G$ a simple graph.

Output: TruE if $G$ is a complete intersection or FALSE otherwise

$H:=G ; \mathfrak{B}:=\emptyset$

while $\exists v \in V(H)$ with $\operatorname{deg}_{H}(v) \leq 2$ do

if $\operatorname{deg}_{H}(v)=2$ and $b(H \backslash\{v\})=b(H)$ then

$W:=\{v\} \cup N_{H}(v) \cup\{u \in V(H) \mid b(H \backslash\{u, v\})>b(H \backslash\{u\})\}$

if not exists an even closed walk such that $V(w)=W$ then

return FALSE

\section{end if}

Let $w$ be a shortest even closed walk with $V(w)=W$.

$\mathfrak{B}:=\mathfrak{B} \cup\left\{B_{w}\right\}$

end if

$H:=H \backslash\{v\}$

\section{end while}

Let $H_{1}, \ldots, H_{s}$ be the connected components of $H$

if exists $i$ such that $H_{i}$ is not odd band or even Möbius band then return FALSE

end if

Let $\mathfrak{B}_{i}$ be a minimal set of generators of $P_{H_{i}}$ for $1 \leq i \leq s$.

if $P_{G}=\left\langle\mathfrak{B} \cup \mathfrak{B}_{1} \cup \cdots \cup \mathfrak{B}_{s}\right\rangle$ then

return TRUE

end if

return FALSE

Figure 5: Pseudo-code for checking whether a graph is a complete intersection. It returns TrUE if $G$ is a complete intersection and FaLSE otherwise.

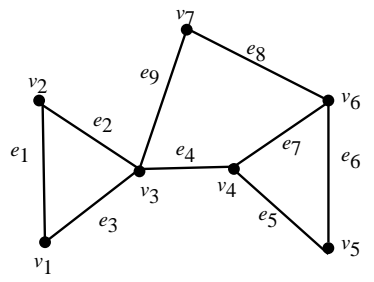

Figure 6: A non complete intersection graph. 
Now we consider the graph $H:=G \backslash\left\{v_{7}\right\}$ and observe that $\operatorname{deg}_{H}\left(v_{1}\right)=2$ and $b(H)=0=b\left(H \backslash\left\{v_{1}\right\}\right)$. We set $W_{2}:=\left\{v_{1}\right\} \cup N_{H}\left(v_{1}\right) \cup\left\{u \in V(H) \mid b\left(H \backslash\left\{u, v_{1}\right\}\right)>b(H \backslash\{u\})\right\}=V(H)$. We construct $w_{2}$ a shortest even closed walk with $V\left(w_{2}\right)=W_{2}$. Then $w_{2}=\left(C_{1}, \mathcal{P}_{1}, C_{2},-\mathcal{P}_{1}\right)$ where $C_{1}:=\left(v_{3}, v_{1}, v_{2}, v_{3}\right)$ and $C_{2}:=\left(v_{4}, v_{5}, v_{6}, v_{4}\right)$ are odd cycles and $\mathcal{P}_{1}$ is the length one path $\mathcal{P}_{1}:=\left(v_{3}, v_{4}\right)$; thus $B_{w_{2}}=x_{1} x_{4}^{2} x_{6}-x_{2} x_{3} x_{5} x_{7}$.

Hence, we consider the graph $H^{\prime}:=H \backslash\left\{v_{1}\right\}$. We observe that every $u \in V\left(H^{\prime}\right), u \neq v_{4}$ either has degree 1 or has degree 2 and $b\left(H^{\prime}\right) \neq b\left(H^{\prime} \backslash\{u\}\right)$. Thus, one can remove one by one every vertex of $H^{\prime}$ until getting a trivial graph.

Then, we have that $G$ is a complete intersection if and only if $P_{G}=\left(B_{w_{1}}, B_{w_{2}}\right)$. We denote by $B$ the $2 \times 9$ matrix $B:=$ $\left(\begin{array}{ccccccccc}0 & 0 & 0 & 1 & 0 & 0 & -1 & 1 & -1 \\ 1 & -1 & -1 & 2 & -1 & 1 & -1 & 0 & 0\end{array}\right)$, then $\Delta_{2}(B)=1$ and it has a square mixed submatrix $B^{\prime}:=\left(\begin{array}{cc}1 & -1 \\ 2 & -1\end{array}\right)$. Thus $P_{G}$ is not a complete intersection.

\section{Theta graphs and complete intersections}

This section is devoted to prove that if $G$ is a complete intersection and there are three paths $\mathcal{P}_{1}, \mathcal{P}_{2}$ and $\mathcal{P}_{3}$ of the same parity connecting $x, y \in V(G)$ that only meet at their ends, i.e., $V\left(\mathcal{P}_{i}\right) \cap V\left(\mathcal{P}_{j}\right)=\{x, y\}$ for $1 \leq i<j \leq 3$, then $\mathcal{P}_{1}, \mathcal{P}_{2}$ and $\mathcal{P}_{3}$ are all odd paths and $\{x, y\} \in E(G)$. We will prove this result in Theorem 5.7 To prove this we will first introduce two results concerning the vertices of degree 2 in a complete intersection graph, namely Lemma 5.1 and Proposition 5.3 The first one is a technical lemma which will very useful in the sequel. The second one describes an operation in $G$ that leads to another graph $G^{\prime}$ with less vertices and edges than $G$ and $G^{\prime}$ is a complete intersection whenever $G$ is.

Lemma 5.1. Let $v$ be a vertex of degree 2 and $H_{1}, H_{2}$ two induced subgraphs such that $v \in V\left(H_{i}\right), \operatorname{deg}_{H_{i}}(v)=2$ and $b\left(H_{i} \backslash\{v\}\right)=b\left(H_{i}\right)$ for $i=1,2$. If $G$ is a complete intersection, then $b(H \backslash\{v\})=b(H)$, where $H:=\left[V\left(H_{1}\right) \cap V\left(H_{2}\right)\right]$.

Proof. Let $\left\{B_{w_{1}}, \ldots, B_{w_{r}}\right\}$ be a minimal set of generators of $P_{G}$ where $w_{j}$ is an even closed walk for $1 \leq j \leq r$. For every $G^{\prime} \in\left\{G, H_{1}, H_{2}, H\right\}$, by Proposition 3.3 and Theorem 3.4 we have that $G^{\prime}$ and $G^{\prime} \backslash\{v\}$ are complete intersections minimally generated by $\left\{B_{w_{i}} \mid V\left(w_{i}\right) \subset V\left(G^{\prime}\right)\right\}$ and $\left\{B_{w_{i}} \mid v \notin V\left(w_{i}\right) \subset V\left(G^{\prime}\right)\right\}$, respectively. Thus,

$$
\begin{gathered}
1+b\left(G^{\prime}\right)-b\left(G^{\prime} \backslash\{v\}\right)=\operatorname{deg}_{G^{\prime}}(v)-1+b\left(G^{\prime}\right)-b\left(G^{\prime} \backslash\{v\}\right)= \\
=\operatorname{ht}\left(P_{G^{\prime}}\right)-\operatorname{ht}\left(P_{G^{\prime} \backslash\{v\}}\right)=\left|\left\{i \mid v \in V\left(w_{i}\right) \subset V\left(G^{\prime}\right)\right\}\right| .
\end{gathered}
$$

In particular, for $i=1,2$ we have that there exists a unique $j_{i} \in\{1, \ldots, r\}$ such that $v \in V\left(w_{j_{i}}\right) \subset V\left(H_{i}\right)$. We claim that $j_{1}=j_{2}$, indeed $v \in V\left(w_{j_{1}}\right) \cap V\left(w_{j_{2}}\right)$ and $1 \geq 1+b(G)-b(G \backslash\{v\})=\left|\left\{i \mid v \in V\left(w_{i}\right)\right\}\right|$. Thus, $V\left(w_{j_{1}}\right) \subset V\left(H_{1}\right) \cap V\left(H_{2}\right)=$ $V(H)$ and we can conclude that $1+b(H)-b(H \backslash\{v\})=\left|\left\{j \mid v \in V\left(w_{j}\right) \subset V(H)\right\}\right|=1$, and $b(H)=b(H \backslash\{v\})$.

The second result concerns an operation in a graph, which is called the contraction of a graph in a vertex of degree 2. We will prove that if $G$ is a complete intersection, the contraction of $G$ in a vertex of degree 2 preserves the property of being a complete intersection.

Definition 5.2. Let $G$ be a graph with a vertex $v$ of degree 2 which does not belong to a triangle, i.e., $N_{G}(v)=\left\{u_{1}, u_{2}\right\}$ and $\left\{u_{1}, u_{2}\right\} \notin E(G)$. We define the contraction of $G$ in $v$ as the graph $G_{v}^{c}$ obtained by contracting the two edges incident to $v$. More precisely, $G_{v}^{c}$ is the graph with

$$
\begin{gathered}
V\left(G_{v}^{c}\right):=\left(V(G) \backslash\left\{v, u_{1}, u_{2}\right\}\right) \cup\{u\} \text { and } \\
E\left(G_{v}^{c}\right):=E\left(G \backslash\left\{v, u_{1}, u_{2}\right\}\right) \cup\left\{\{u, x\} \mid\left\{u_{1}, x\right\} \text { or }\left\{u_{2}, x\right\} \in E(G) \text { and } x \neq v\right\} .
\end{gathered}
$$


Proposition 5.3. Let $G$ be a graph with a vertex $v$ of degree 2 which does not belong to a triangle. If $G$ is a complete intersection, then so is $G_{v}^{c}$.

Proof. For every even (respect. odd) closed walk $w=\left(z_{1}, \ldots, z_{r}=z_{1}\right)$ in $G$, we define $\widehat{w}$ as the even (respect. odd) closed walk in $G_{v}^{c}$ constructed as follows. Assume that $z_{1} \notin\left\{v, u_{1}, u_{2}\right\}$, for every $i \in\{2, \ldots, r-1\}$ such that $z_{i}=v$, then $z_{i-1}, z_{i+1} \in\left\{u_{1}, u_{2}\right\}$ and we set $\widehat{w}:=\left(z_{1}, \ldots, z_{i-2}, u, z_{i+2}, \ldots, z_{r}=x_{1}\right)$ and whenever $z_{i} \in\left\{u_{1}, u_{2}\right\}$ with $z_{i-1} \neq v, z_{i+1} \neq v$ then we set $\widehat{w}:=\left(z_{1}, \ldots, z_{i-1}, u, z_{i+1}, \ldots, z_{r}=z_{1}\right)$. Note that it might happen that $w$ passes by $u_{1}, u_{2}$ or $v$ more than once. Moreover, for every closed walk $w^{\prime}$ in $G_{v}^{c}$ one can find another $w$ in $G$ such that $w^{\prime}=\widehat{w}$.

We have that $G$ is bipartite if and only if so is $G_{v}^{c}$, indeed $V_{1}, V_{2}$ is a bipartition for $G$ with $u_{1} \in V_{1}$ if and only if $V_{1}^{\prime}, V_{2}^{\prime}$ is a bipartition for $G_{v}^{c}$, where $u \in V_{1}^{\prime}, V_{1} \backslash\left\{u_{1}, u_{2}\right\}=V_{1}^{\prime} \backslash\{u\}$ and $V_{2} \backslash\{v\}=V_{2}^{\prime}$. Moreover, since $\left|V\left(G_{v}^{c}\right)\right|=m-2$ and $\left|E\left(G_{v}^{c}\right)\right|=n-\left|N_{G}\left(u_{1}\right) \cap N_{G}\left(u_{2}\right)\right|-1$, we have that

$$
\operatorname{ht}\left(P_{G_{v}^{c}}\right)=\operatorname{ht}\left(P_{G}\right)-\left|N_{G}\left(u_{1}\right) \cap N_{G}\left(u_{2}\right)\right|+1 .
$$

Moreover $\left|N_{G}\left(u_{1}\right) \cap N_{G}\left(u_{2}\right)\right| \leq 2$, otherwise $G$ has a subgraph $\mathcal{K}_{2,3}$, which is not possible by Corollary 3.9 . This proof falls naturally into two parts.

If $N_{G}\left(u_{1}\right) \cap N_{G}\left(u_{2}\right)=\{v\}$. Assume that $e_{n-1}=\left\{u_{1}, v\right\}, e_{n}=\left\{u_{2}, v\right\}$ and set $e_{i}^{\prime}:=e_{i}$ if $e_{i} \in E\left(G_{v}^{c}\right)$ and $e_{i}^{\prime}:=\{u, z\}$ if either $e_{i}=\left\{u_{1}, z\right\}$ or $e_{i}=\left\{u_{2}, z\right\}$ for every $1 \leq i \leq n-2$; then $E\left(G_{v}^{c}\right)=\left\{e_{1}^{\prime}, \ldots, e_{n-2}^{\prime}\right\}$.

Consider now the morphism $\psi: k\left[x_{1}, \ldots, x_{n}\right] \longrightarrow k\left[x_{1}, \ldots, x_{n-2}\right]$ induced by $x_{n-1} \mapsto 1, x_{n} \mapsto 1$ and $x_{i} \mapsto x_{i}$ for every $i \in\{1, \ldots, n-2\}$. It is easy to check that for every even closed walk $w$ in $G$, then $\psi\left(B_{w}\right)=B_{\widehat{w}}$. This implies that $\psi\left(P_{G}\right)=P_{G_{v}^{c}}$. Since ht $\left(P_{G}\right)=\operatorname{ht}\left(P_{G_{v}^{c}}\right)$, we get that $G_{v}^{c}$ is a complete intersection because if $\mathfrak{B}$ is a set of generators of $P_{G}$ then $\psi(\mathfrak{B})$ is a set of generators of $P_{G_{v}^{c}}$.

Secondly consider the case where $N_{G}\left(u_{1}\right) \cap N_{G}\left(u_{2}\right)=\{v, z\}$. Suppose that $e_{n-3}=\left\{u_{1}, z\right\}, e_{n-2}=\left\{u_{2}, z\right\}, e_{n-1}=\left\{u_{2}, v\right\}$ and $e_{n}=\left\{u_{1}, v\right\}$ and set $e_{i}^{\prime}:=e_{i}$ if $e_{i} \in E\left(G_{v}^{c}\right), e_{i}^{\prime}:=\{u, t\}$ if either $e_{i}=\left\{u_{1}, t\right\}$ or $e_{i}=\left\{u_{2}, t\right\}$ for all $i \in\{1, \ldots, n-4\}$ and $e_{n-3}^{\prime}:=\{u, z\}$; then $E\left(G_{v}^{c}\right)=\left\{e_{1}^{\prime}, \ldots, e_{n-3}^{\prime}\right\}$.

Consider now the morphism $\psi: k\left[x_{1}, \ldots, x_{n}\right] \longrightarrow k\left[x_{1}, \ldots, x_{n-3}\right]$ defined by $x_{n-2} \mapsto x_{n-3}, x_{n-1} \mapsto 1, x_{n} \mapsto 1$ and $x_{i} \mapsto x_{i}$ for every $i \in\{1, \ldots, n-3\}$. Proceeding as before, we get that $\psi\left(P_{G}\right)=P_{G_{v}^{c}}$.

Suppose that $G$ is a complete intersection and consider the cycle $w:=\left(v, u_{1}, z, u_{2}, v\right)$, then the quadric $B_{w}:=$ $x_{n-2} x_{n}-x_{n-3} x_{n-1} \in P_{G}$. Since $P_{G}$ is a homogeneous ideal which does not contain any linear form, we get that there exists a minimal set of generators $\mathfrak{B}$ of $P_{G}$ such that $\mathfrak{B}=\left\{B_{w_{1}}, \ldots, B_{w_{r}}, B_{w}\right\}$ for some even closed walks $w_{1}, \ldots, w_{r}$ in $G$ and then $r+1=\operatorname{ht}\left(P_{G}\right)$. Since $\psi\left(P_{G}\right)=P_{G_{v}^{c}}$ and $\psi\left(B_{w}\right)=0$, it follows that $\left\{\psi\left(B_{w_{1}}\right), \psi\left(B_{w_{2}}\right), \ldots, \psi\left(B_{w_{r}}\right)\right\}$ generates $P_{G_{v}^{c}}$ and ht $\left(P_{G_{v}^{c}}\right)=r$, thus it is a complete intersection.

The converse of Proposition 5.3 is not true in general, as one can see in Figure 7
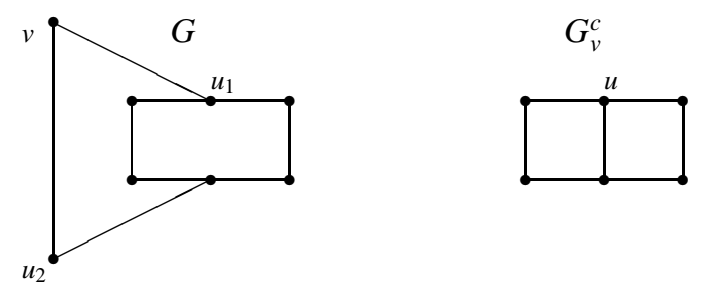

Figure 7: $G$ is bipartite but it is not a ring graph, hence it is not a complete intersection. Nevertheless, $v$ is a vertex of degree 2 which does not belong to a triangle and $G_{v}^{c}$ is a complete intersection.

Now we introduce the concept of theta graph and use the previous results to prove Theorem 5.7, which asserts that odd theta graph whose base vertices are not adjacent, and also even theta graphs, are forbidden subgraphs in a complete intersection graph. This is the main result of this section. 
Definition 5.4. A theta graph $T$ with base vertices $x, y$ is a graph with $V(T):=V\left(\mathcal{P}_{1}\right) \cup V\left(\mathcal{P}_{2}\right) \cup V\left(\mathcal{P}_{3}\right)$, where $\mathcal{P}_{1}, \mathcal{P}_{2}$ and $\mathcal{P}_{3}$ are three paths of length $\geq 2$ connecting $x$ and $y$ such that $V\left(\mathcal{P}_{i}\right) \cap V\left(\mathcal{P}_{j}\right)=\{x, y\}$ for $1 \leq i<j \leq 3$. If $\mathcal{P}_{1}, \mathcal{P}_{2}$ and $\mathcal{P}_{3}$ are all even (respect. odd) paths, then $T$ is called an even (respect. odd) theta graph.

Remark 5.5. Theta graphs are sometimes defined in the literature to have $E(T)=E\left(\mathcal{P}_{1}\right) \cup E\left(\mathcal{P}_{2}\right) \cup E\left(\mathcal{P}_{3}\right)$. However, from our definition we have $E\left(\mathcal{P}_{1}\right) \cup E\left(\mathcal{P}_{2}\right) \cup E\left(\mathcal{P}_{3}\right) \subset E(T)$; this is, it might have edges connecting a vertex in $\mathcal{P}_{i}$ and a vertex in $\mathcal{P}_{j}$ with $1 \leq i<j \leq 3$ or even connecting two vertices $a_{i}, a_{j}$ of $\mathcal{P}_{k}=\left(x=a_{0}, a_{1}, \ldots, a_{r-1}, a_{r}=y\right)$ with $0 \leq i<i+1<j \leq r$ and $1 \leq k \leq 3$.

To prove Theorem 5.7 we need a lemma, whose proof is almost immediate.

Lemma 5.6. Let $v$ be a vertex of degree 2 and $C$ an even cycle such that $v \in V(C)$. Then, $G$ is bipartite $\Longleftrightarrow G \backslash\{v\}$ is bipartite.

Proof. Let $u_{1}, u_{2}$ be the neighbors of $v$, then $u_{1}, u_{2} \in V(C)$. Hence, if $V_{1}, V_{2}$ is a bipartition for $G \backslash\{v\}$ and $u_{1} \in V_{1}$, then necessarily $u_{2} \in V_{1}$, so $V_{1}, V_{2} \cup\{v\}$ is a bipartition for $G$. The other implication is obvious.

Theorem 5.7. Neither odd theta graphs whose base vertices are not adjacent nor even theta graphs are complete intersections.

Proof. Suppose that there exists an even theta graph or an odd theta graph whose base vertices are not adjacent which is a complete intersection. Let $G$ be the smallest graph with respect to $|V(G)|$ with this property, we denote by $x, y$ the base vertices of $G$ and $\mathcal{P}_{1}, \mathcal{P}_{2}, \mathcal{P}_{3}$ the three even or odd paths connecting $x$ and $y$ such that $V\left(\mathcal{P}_{i}\right) \cap V\left(\mathcal{P}_{j}\right)=\{x, y\}$ for $1 \leq i<j \leq 3$.

If $G$ is 3-regular, then one can write $N_{G}(x)=\left\{u_{1}, u_{2}, u_{3}\right\}$ with $u_{i} \in V\left(\mathscr{P}_{i}\right)$ and we claim that $b\left(G \backslash\left\{u_{i}\right\}\right)=b\left(G \backslash\left\{x, u_{i}\right\}\right)$ for $1 \leq i \leq 3$. Indeed, we can suppose that $i=1$, then $x$ has degree 2 in $G \backslash\left\{u_{1}\right\}$ and belongs to the even cycle $\left(\mathcal{P}_{2},-\mathcal{P}_{3}\right)$; then by Lemma 5.6 we have that $b\left(G \backslash\left\{u_{1}\right\}\right)=b\left(G \backslash\left\{x, u_{1}\right\}\right)$. On the other hand, since $G$ is a complete intersection 3-regular graph, it is an odd band or an even Möbius band. Thus one can write $V(G)=\left\{a_{1}, \ldots, a_{r}, b_{1}, \ldots, b_{r}\right\}$ and assume that $x=a_{i}$ for some $1 \leq i \leq r$. Then $b\left(G \backslash\left\{b_{i}\right\}\right)=0$ and $b\left(G \backslash\left\{x, b_{i}\right\}\right)=1$, which is a contradiction.

Then, by Corollary 3.8 there exists $v \in V(G)$ of degree 2 and we will assume that $v \in V\left(\mathcal{P}_{3}\right)$. Then we consider $C_{i}:=\left(\mathcal{P}_{i},-\mathcal{P}_{3}\right)$ and $H_{i}:=\left[V\left(C_{i}\right)\right]$ for $i=1,2$. Since $C_{i}$ is an even cycle and $v \in V\left(C_{i}\right)$, it follows by Lemma 5.6 that $b\left(H_{i}\right)=b\left(H_{i} \backslash\{v\}\right)$ for $i=1,2$. Thus by Lemma5.1, $b(H)=b(H \backslash\{v\})$ where $H:=\left[V\left(H_{1}\right) \cap V\left(H_{2}\right)\right]=\left[V\left(\mathcal{P}_{3}\right)\right]$, let us prove that this is not possible. We denote $\mathcal{P}_{3}:=\left(x=a_{0}, a_{1}, \ldots, a_{r}=y\right)$, then $v=a_{i}$ for some $1 \leq i<r$.

If $r=2$, then $v=a_{1}$. Moreover, if $\left\{a_{0}, a_{2}\right\} \in E(G)$ we have that $b(H)=0 \neq 1=b(H \backslash\{v\})$ and if $\left\{a_{0}, a_{2}\right\} \notin E(G)$ we have that $b(H)=1 \neq 2=b(H \backslash\{v\})$.

If $r=3$, then $G$ is an odd theta graph and $\{x, y\} \notin E(G)$, we can assume that $v=a_{1}$, then if $\left\{a_{0}, a_{2}\right\} \in E(G)$ we have that $b(H)=0 \neq 1=b(H \backslash\{v\})$ and if $\left\{a_{0}, a_{2}\right\} \notin E(G)$ we have that $b(H)=1 \neq 2=b(H \backslash\{v\})$.

If $r \geq 4$, let us prove that $\left\{a_{i-1}, a_{i+1}\right\} \in E(G)$. Assume that $\left\{a_{i-1}, a_{i+1}\right\} \notin E(G)$ and consider $G_{v}^{c}$, which is an even or odd theta graph. By Proposition 5.3, $G_{v}^{c}$ is a complete intersection and $\left|V\left(G_{v}^{c}\right)\right|<|V(G)|$. This contradicts the minimality of $G$ unless if $G_{v}^{c}$ is an odd theta graph whose base vertices are adjacent. This can only happen if $v=a_{1}$ and $\left\{a_{2}, y\right\} \in E(G)$, or $v=a_{r}$ and $\left\{a_{r-1}, x\right\} \in E(G)$. Without loss of generality we can assume that $v=a_{1}$ and $\left\{a_{2}, y\right\} \in E(G)$. Then, the induced subgraph $G^{\prime}:=\left[V\left(\mathcal{P}_{1}\right) \cup V\left(\mathcal{P}_{2}\right) \cup V\left(\mathcal{P}_{3}^{\prime}\right)\right]$ with $\mathcal{P}_{3}^{\prime}=\left(x, a_{1}, a_{2}, y\right)$ is an odd theta graph whose base vertices $x, y$ are not adjacent and is a complete intersection, but this is not possible by the minimality of $G$. Thus, $\left\{a_{i-1}, a_{i+1}\right\} \in E(G)$. 
Since $\left\{a_{i-1}, a_{i+1}\right\} \in E(G)$, then $b(H)=0$; let us see that $H \backslash\{v\}$ is bipartite and $b(H \backslash\{v\})=1$. Indeed, suppose that $H \backslash\{v\}$ is not bipartite, we denote $a_{j}^{\prime}:=a_{j}$ for $1 \leq j \leq i-1, a_{j}^{\prime}:=a_{j+1}$ for $i \leq j<r$. Since $\left\{a_{j}^{\prime}, a_{j+1}^{\prime}\right\} \in E(H \backslash\{v\})$ for $1 \leq j<r$, there exists $1 \leq j<k<r$ such that $\left\{a_{j}^{\prime}, a_{k}^{\prime}\right\} \in E(H)$ and $j \equiv k(\bmod 2)$. We separate three cases:

(a) if $k<i$, then $\left\{a_{j}, a_{k}\right\} \in E(H)$.

(b) if $j \geq i$, then $\left\{a_{j+1}, a_{k+1}\right\} \in E(H)$

(c) $j<i \leq k$, then $\left\{a_{j}, a_{k+1}\right\} \in E(H)$.

If (a) holds, we denote $\mathcal{P}_{3}^{\prime}=\left(x=a_{0}, \ldots, a_{j}, a_{k}, \ldots, a_{i-1}, a_{i+1}, \ldots, a_{r}=y\right)$. If (b) holds, we denote $\mathcal{P}_{3}^{\prime}=(x=$ $\left.a_{0}, \ldots, a_{i-1}, a_{i+1}, \ldots, a_{j+1}, a_{k+1}, \ldots, a_{r}=y\right)$. If (c) holds, we denote $\mathcal{P}_{3}^{\prime}=\left(x=a_{0}, \ldots, a_{j}, a_{k+1}, \ldots, a_{r}=y\right)$. In the three cases $\mathcal{P}_{3}^{\prime}$ is an walk of the same parity of $\mathcal{P}_{3}$ connecting $x$ and $y$ with $V\left(\mathcal{P}_{3}^{\prime}\right) \subsetneq V\left(\mathcal{P}_{3}\right)$, but this contradicts again the minimality of $G$.

\section{Structure theorems for complete intersection graphs}

The goal of this section is to prove two structure theorems for complete intersection connected graphs; namely Theorem 6.5 and Theorem 6.18 Given a graph, it can be partitioned into two induced subgraphs $C$ and $R$, such that $V(C)=V\left(C_{1}\right) \sqcup \cdots \sqcup V\left(C_{S}\right)$ where $C_{1}, \ldots, C_{s}$ are odd primitive cycles and $R$ is a bipartite graph. Note that this partition might not be unique and when $G$ is bipartite, one has that $C$ is the empty graph. Whenever we have a partition with these properties we write $G=[C ; R]$. In order to characterize the complete intersection property on $G$, we propose to characterize when $C$ and $R$ are complete intersections, and then determine the admissible edges connecting $C$ and $R$. Since $R$ is a bipartite graph it turns out that it is a complete intersection if and only if it is a ring graph (see [11, Corollary 3.3]). Theorem 6.5 will give necessary conditions for a connected graph to be a complete intersection by determining when $C$ is a complete intersection. Finally, if $C$ is connected and $R$ is 2-connected, Theorem 6.18 characterizes the complete intersection property by obtaining all possible edges connecting $C$ and $R$.

Let us start with this proposition.

Proposition 6.1. Let $G$ be a complete intersection connected graph, then there are at most two vertex disjoint odd cycles in $G$.

We need the following technical result which is included in the proof of [28, Theorem 5.3]. Recall that a block is a maximal connected subgraph $B$ of $G$ such that if one removes any of its vertices it is still connected. A graph is 2 -connected if it only has one block and more than 2 vertices.

Lemma 6.2. Let $G$ be a complete intersection 2-connected graph and let $C_{1}, C_{2}$ be two odd cycles in $G$.

(a) If $V\left(C_{1}\right) \cap V\left(C_{2}\right)=\{v\}$, then there exists an $e \in E(G)$ such that $v \notin e$ and $e \cap V\left(C_{i}\right) \neq \emptyset$ for $i \in\{1,2\}$.

(b) If $C_{1}$ and $C_{2}$ are vertex disjoint, then there exist $e_{1}, e_{2} \in E(G)$ such that $e_{1} \cap e_{2}=\emptyset$ and $e_{i} \cap V\left(C_{j}\right) \neq \emptyset$ for $i, j \in\{1,2\}$.

Proof of Proposition 6.1. Assume that $G$ is a complete intersection with three vertex disjoint odd cycles and let $G^{\prime}$ be the smallest connected induced subgraph with this property. We denote by $C_{1}=\left(a_{1}, \ldots, a_{r_{1}}, a_{1}\right), C_{2}=\left(b_{1}, \ldots, b_{r_{2}}, b_{1}\right)$ and $C_{3}=\left(c_{1}, \ldots, c_{r_{3}}, c_{1}\right)$ three vertex disjoint odd primitive cycles of $G^{\prime}$. By [28, Theorem 4.2], $G^{\prime}$ has either one or two non bipartite blocks and the proof falls naturally in two cases. 
If $G^{\prime}$ has only one non bipartite block, then $C_{1}, C_{2}$ and $C_{3}$ belong to it and, by Lemma 6.2 , for every $1 \leq i<j \leq 3$ there exist two edges connecting a vertex of $C_{i}$ and a vertex of $C_{j}$; thus $G^{\prime}=\left[V\left(C_{1}\right) \cup V\left(C_{2}\right) \cup V\left(C_{3}\right)\right]$. $G^{\prime}$ can not be a band or a Möbius band because there are three vertex disjoint odd primitive cycles, then there exists a vertex $z \in V\left(G^{\prime}\right)$ of degree 2. Suppose that $z \in V\left(C_{3}\right)$ and denote by $H_{i}:=\left[V\left(C_{i}\right) \cup V\left(C_{3}\right)\right]$ for $i=1,2$. We have that $H_{i} \backslash\{z\}$ is connected and $V\left(C_{i}\right) \subset V\left(H_{i} \backslash\{z\}\right)$, then $b\left(H_{i}\right)=b\left(H_{i} \backslash\{z\}\right)=0$ for $i=1,2$. By Lemma 5.1 we have that $b(H)=b(H \backslash\{z\})$ where $H:=\left[V\left(H_{1}\right) \cap V\left(H_{2}\right)\right]=\left[V\left(C_{3}\right)\right]$. However, $C_{3}$ is an odd primitive cycle, then $b(H)=0$ and $b(H \backslash\{z\})=1$, which is a contradiction.

If $G^{\prime}$ has two non bipartite blocks, then two of the odd cycles belong to the same block of $G^{\prime}$, say $C_{1}$ and $C_{2}$. By Lemma 6.2, $C_{1}$ and $C_{2}$ are connected by at least two edges. Moreover, $C_{3}$ is not in the same block of $C_{1}$ and $C_{2}$. Then we set $G_{1}:=\left[V\left(C_{1}\right) \cup V\left(C_{2}\right)\right]$ and take $\mathcal{P}$ a path in $G^{\prime}$ of minimum length connecting a vertex of $C_{3}$ and a vertex of $G_{1}$. By the minimality of $G^{\prime}$ we have that $G^{\prime}=\left[V\left(G_{1}\right) \cup V(\mathcal{P}) \cup V\left(C_{3}\right)\right]$. Moreover, we can assume that there exists $s \geq 0$ such that $\mathcal{P}=\left(c_{1}=u_{0}, u_{1}, \ldots, u_{s}, a_{1}\right)$; since $\mathcal{P}$ has minimum length one can deduce that $u_{i} \notin V\left(G_{1}\right) \cup V\left(C_{3}\right)$ for $1 \leq i \leq s,\left\{c_{j}, u_{i}\right\} \notin E\left(G^{\prime}\right)$ for every $1 \leq j \leq r_{3}, i>1$ and $\left\{a_{j}, u_{i}\right\} \notin E\left(G^{\prime}\right)$ for every $1 \leq j \leq r_{1}, i<s$.

Firstly assume that $\operatorname{deg}_{G^{\prime}}\left(c_{j}\right)=2$ for every $j>1$ and take $u:=c_{2}$. We set $\mathcal{P}^{\prime}$ the shortest path in $G^{\prime} \operatorname{connecting} c_{1}$ with a vertex of $C_{2}$. Then, we can assume that $\mathcal{P}^{\prime}=\left(c_{1}=v_{0}, v_{1}, \ldots, v_{t}=b_{1}\right)$ and have that

(a) $v_{i}=u_{i}$ for $0 \leq i \leq s, v_{i} \in V\left(C_{1}\right)$ for $s<i \leq t-1$ and $v_{t} \in V\left(C_{2}\right)$,

(b) $\left\{v_{i}, v_{j}\right\} \notin E\left(G^{\prime}\right)$ for $0 \leq i<i+1<j \leq t$.

Now we set $H_{1}:=\left[V\left(C_{1}\right) \cup V(\mathcal{P}) \cup V\left(C_{3}\right)\right]$ and $H_{2}:=\left[V\left(C_{2}\right) \cup V\left(\mathcal{P}^{\prime}\right) \cup V\left(C_{3}\right)\right]$; clearly $b\left(H_{i}\right)=b\left(H_{i} \backslash\{u\}\right)=0$ for $i=1,2$. However, if we set $H:=\left[V\left(H_{1}\right) \cap V\left(H_{2}\right)\right]=\left[V\left(C_{3}\right) \cup\left\{v_{1}, \ldots, v_{t-1}\right\}\right]$, then $V\left(C_{3}\right) \subset V(H), \operatorname{deg}_{H}\left(c_{1}\right)=$ $3, \operatorname{deg}_{H}\left(v_{t-1}\right)=1$ and $\operatorname{deg}_{H}(v)=2$ for the rest of vertices of $H$. Thus $b(H)=0$ and $b(H \backslash\{u\})=1$, which contradicts Lemma 5.1

So assume that $\operatorname{deg}_{G^{\prime}}\left(c_{j}\right)>2$ for some $j>1$ and let us see that $s=0$; i.e., $\left\{a_{1}, c_{1}\right\} \in E\left(G^{\prime}\right)$. Indeed, if $s \geq 1$, by the minimality of $G^{\prime}$ we have that $\left\{u_{1}, c_{j}\right\} \in E\left(G^{\prime}\right)$, but then there exists an odd primitive cycle $C^{\prime}$ such that $u_{1} \in V\left(C^{\prime}\right) \subset V\left(C_{3}\right) \cup\left\{u_{1}\right\}$, which contradicts the minimality of $G^{\prime}$. Thus, $s=0,\left\{c_{1}, a_{1}\right\} \in E\left(G^{\prime}\right)$ and if $\left\{c_{k}, a_{i}\right\} \in E\left(G^{\prime}\right)$ then $i=1$ because $G^{\prime}$ has two blocks. Let $C_{3}^{\prime}$ be an odd primitive cycle such that $a_{1} \in V\left(C_{3}^{\prime}\right) \subset V\left(C_{3}\right) \cup\left\{a_{1}\right\}$ and take $u \in V\left(C_{3}^{\prime}\right), u \neq a_{1}$. We set $\mathcal{P}^{\prime}$ the shortest path in $G^{\prime}$ connecting $a_{1}$ with a vertex of $C_{2}$. Then, we can assume that $\mathcal{P}^{\prime}=\left(a_{1}=v_{0}, v_{1}, \ldots, v_{t}=b_{1}\right)$ and we have that

(a) $v_{i} \in V\left(C_{1}\right)$ for $0 \leq i \leq t-1$

(b) $\left\{v_{i}, v_{j}\right\} \notin E\left(G^{\prime}\right)$ for $0 \leq i<i+1<j \leq t$.

Now we set $H_{1}:=\left[V\left(C_{1}\right) \cup V\left(C_{3}^{\prime}\right)\right]$ and $H_{2}:=\left[V\left(C_{2}\right) \cup V\left(\mathcal{P}^{\prime}\right) \cup V\left(C_{3}^{\prime}\right)\right]$; and have that $u$ has degree 2 in $\left[V\left(H_{1}\right) \cup V\left(H_{2}\right)\right]$ and clearly $\operatorname{deg}_{H_{i}}(u)=2, b\left(H_{i}\right)=b\left(H_{i} \backslash\{u\}\right)=0$ and $\operatorname{deg}_{H_{i}}(u)=2$ for $i=1,2$. However, if we set $H:=\left[V\left(H_{1}\right) \cap V\left(H_{2}\right)\right]=\left[V\left(C_{3}^{\prime}\right) \cup\left\{v_{1}, \ldots, v_{t-1}\right\}\right]$, then $V\left(C_{3}^{\prime}\right) \subset V(H), \operatorname{deg}_{H}\left(a_{1}\right)=3, \operatorname{deg}_{H}\left(v_{t-1}\right)=1 \operatorname{and~deg}_{H}(v)=2$ for the rest of vertices of $H$. Thus $b(H)=0$ and $b(H \backslash\{u\})=1$, which again contradicts Lemma 5.1.

Now that we know that there are at most two vertex disjoint odd primitive cycles in a complete intersection connected graph, let us determine how two such cycles can be connected.

Definition 6.3. A graph $G$ is called an odd partial band if there exist two vertex disjoint odd primitive cycles $C_{1}=$ $\left(a_{1}, \ldots, a_{r_{1}}, a_{1}\right)$ and $C_{2}=\left(b_{1}, \ldots, b_{r_{2}}, b_{1}\right)$ such that $V(G)=V\left(C_{1}\right) \cup V\left(C_{2}\right)$ and

$$
E(G)=E\left(C_{1}\right) \cup E\left(C_{2}\right) \cup\left\{\left\{a_{j_{1}}, b_{k_{1}}\right\}, \ldots,\left\{a_{j_{s}}, b_{k_{s}}\right\}\right\},
$$

where $s \geq 1,1 \leq j_{1} \leq \cdots \leq j_{s} \leq r_{1}, 1 \leq k_{1} \leq \cdots \leq k_{s} \leq r_{2}$ and $j_{i} \equiv k_{i}(\bmod 2)$ (see Figure 8). 


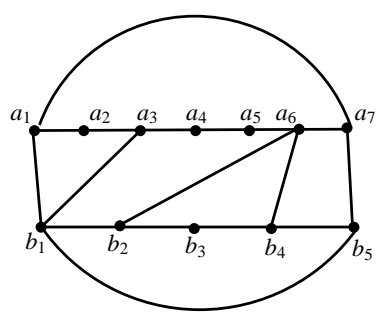

Figure 8: An odd partial band

Proposition 6.4. Let $G$ be a connected graph with $V(G)=V\left(C_{1}\right) \cup V\left(C_{2}\right)$ where $C_{1}$ and $C_{2}$ are two vertex disjoint odd primitive cycles. Then, $G$ is a complete intersection if and only if $G$ is an odd partial band.

Proof. $(\Rightarrow)$ We will proceed by induction on $|V(G)|$. If $|V(G)|=6$, i.e., $C_{1}$ and $C_{2}$ are triangles, the number of edges has to be less or equal to 9 , because $2|E(G)| \leq 3|V(G)|=18$ by Corollary 3.7. If $7 \leq|E(G)| \leq 8$, then one see at once that $G$ is always an odd partial band. If $|E(G)|=9$, then $G$ is an odd partial band unless if one can write $C_{1}:=\left(a_{1}, a_{2}, a_{3}, a_{1}\right), C_{2}:=\left(b_{1}, b_{2}, b_{3}, b_{1}\right)$ and $E(G)=E\left(C_{1}\right) \cup E\left(C_{2}\right) \cup\left\{e_{1}, e_{2}, e_{3}\right\}$ where $e_{1}=\left\{a_{1}, b_{1}\right\}, e_{2}=\left\{a_{1}, b_{3}\right\}$ and

(a) $e_{3}=\left\{a_{1}, b_{2}\right\}$, or

(b) $e_{3}=\left\{a_{2}, b_{2}\right\}$.

If (a) holds, then $\operatorname{deg}\left(a_{2}\right)=2$ and setting $H_{i}:=\left[\left\{a_{1}, a_{2}, a_{3}, b_{i}, b_{3}\right\}\right]$ we observe that $b\left(H_{i}\right)=b\left(H_{i} \backslash\left\{a_{2}\right\}\right)$ for $i=1,2$. However, setting $H:=\left[V\left(H_{1}\right) \cap V\left(H_{2}\right)\right]=\left[\left\{a_{1}, a_{2}, a_{3}, b_{3}\right\}\right]$, one gets that $b(H)=0$ and $b\left(H \backslash\left\{a_{2}\right\}\right)=1$; which contradicts Lemma 5.1. If (b) holds, then $G$ has a subgraph $\mathcal{K}_{2,3}$ with vertices $\left\{a_{1}, a_{2}, b_{1}, b_{2}, b_{3}\right\}$, a contradiction to Corollary 3.9

Assume now that $|V(G)|>6$. Note that $G$ cannot be an even Möbius band because $G$ has two vertex disjoint odd primitive cycles. Thus, if $\operatorname{deg}(v)>2$ for every $v \in V(G)$, then $G$ is an odd band, which in particular is an odd partial band.

If every $x \in V(G)$ with $\operatorname{deg}(x)=2$ belongs to a triangle, then we can assume that $\operatorname{deg}(y) \geq 3$ for every $y \in$ $V\left(C_{1}\right), C_{1}$ is not a triangle and $C_{2}$ is a triangle. Then $\left|V\left(C_{1}\right)\right| \geq 5$, so $2|E(G)|=\sum_{v \in V(G)} \operatorname{deg}(v)=\sum_{v \in V\left(C_{1}\right)} \operatorname{deg}(v)+$ $\sum_{v \in V\left(C_{2}\right)} \operatorname{deg}(v) \geq 3\left|V\left(C_{1}\right)\right|+2\left|V\left(C_{2}\right)\right|+5>3|V(G)|$, a contradiction to Corollary3.7

We can suppose that there exists $x \in V(G)$ of degree 2 which does not belong to a triangle, we assume that $x \in$ $V\left(C_{1}\right)$. Then, we consider $G_{x}^{c}$ and we have that $V\left(G_{x}^{c}\right)=\left\{a_{1}^{\prime}, \ldots, a_{r_{1}-2}^{\prime}, b_{1}, \ldots, b_{r_{2}}\right\}$, where $C_{1}^{\prime}:=\left(a_{1}^{\prime}, \ldots, a_{r_{1}-2}^{\prime}, a_{1}^{\prime}\right)$ and $C_{2}:=\left(b_{1}, \ldots, b_{r_{2}}, b_{1}\right)$ are odd primitive cycles. Then there exists $2 \leq i \leq r_{1}-1$ such that $x=a_{i}, C_{1}=\left(a_{1}, \ldots, a_{r_{1}}, a_{1}\right)$ and

$$
\left\{a_{j}^{\prime}, b_{k}\right\} \in E\left(G_{x}^{c}\right) \Longleftrightarrow\left\{\begin{array}{ccc}
\left\{a_{j}, b_{k}\right\} \in E(G) & \text { and } & j \leq i-1, \quad \text { or } \\
\left\{a_{j+2}, b_{k}\right\} \in E(G) & \text { and } \quad j \geq i-1 .
\end{array}\right.
$$

By induction hypothesis $G_{x}^{c}$ is an odd partial band. Thus for every $\left\{a_{i}, b_{j}\right\} \in E(G)$ then $i \equiv j(\bmod 2)$, and for every $\left\{a_{i_{1}}, b_{j_{1}}\right\},\left\{a_{i_{2}}, b_{j_{2}}\right\} \in E(G)$ such that $i_{1} \neq i-1$ or $i_{2} \neq i+1$, if $i_{1}<i_{2}$, then $j_{1} \leq j_{2}$. Then, $G$ is an odd partial band unless if there exist two edges $\left\{a_{i-1}, b_{j_{1}}\right\},\left\{a_{i+1}, b_{j_{2}}\right\} \in E(G)$ with $j_{1}>j_{2}$.

If there exist two adjacent vertices in $C_{1}$ of degree 2, then we take $x=a_{i}$ one of these two vertices and there can not exist $\left\{a_{i-1}, b_{j_{1}}\right\},\left\{a_{i+1}, b_{j_{2}}\right\} \in E(G)$ because either $\operatorname{deg}\left(a_{i-1}\right)=2 \operatorname{or} \operatorname{deg}\left(a_{i+1}\right)=2$; hence $G$ is an odd partial band.

If we are not in the previous situation, then there are at least three vertices of degree $\geq 3$ in $C_{1}$. Take $x=a_{i} \in V\left(C_{1}\right)$ a vertex of degree 2 and assume that there exist two edges $\left\{a_{i-1}, b_{j_{1}}\right\},\left\{a_{i+1}, b_{j_{2}}\right\} \in E(G)$ with $j_{1}>j_{2}$, let us prove that 
$G$ is not a complete intersection. Set $u:=b_{j_{2}+1}$, we claim that $\operatorname{deg}(u)=2$. Indeed $\left\{a_{i-1}, u\right\},\left\{a_{i+1}, u\right\} \notin E(G)$ because $j_{2}+1 \not \equiv j_{2} \equiv i-1 \equiv i+1(\bmod 2),\left\{a_{k}, u\right\} \notin E(G)$ if $k<i-1$ because $\left\{a_{i+1}, b_{j_{2}}\right\} \in E(G)$ and $j_{2}<j_{2}+1$; furthermore $\left\{a_{k}, u\right\} \notin E(G)$ if $k>i+1$ because $\left\{a_{i-1}, b_{j_{1}}\right\} \in E(G)$ and $j_{2}+1<j_{1}$.

Take $i^{\prime} \notin\{i-1, i+1\}$ such that $\operatorname{deg}\left(a_{i^{\prime}}\right) \geq 3$, we will assume that $i^{\prime}<i-1$ and set $j^{\prime}:=\max \left\{j \mid\left\{a_{i^{\prime}}, b_{j}\right\} \in E(G)\right\}$. Then necessarily $i^{\prime} \equiv j^{\prime}(\bmod 2)$ and $j^{\prime} \leq j_{2}$. Now we consider the even cycle

$$
w_{1}:=\left(b_{j^{\prime}}, b_{j^{\prime}+1}, \ldots, b_{j_{1}}, a_{i-1}, a_{i-2}, \ldots, a_{i^{\prime}}, b_{j^{\prime}}\right)
$$

and the even closed walk

$$
w_{2}:=\left(b_{j^{\prime}}, b_{j^{\prime}-1}, \ldots, b_{1}, b_{r_{2}}, \ldots, b_{j_{2}}, a_{i+1}, a_{i+2}, \ldots, a_{r_{1}}, a_{1}, \ldots, a_{i^{\prime}}, b_{j^{\prime}}\right),
$$

which consists on an even cycle if $j^{\prime} \neq j_{2}$ or two odd cycles with the vertex $b_{j_{2}}$ in common if $j^{\prime}=j_{2}$. For $i=1,2$ we denote $H_{i}:=\left[V\left(w_{i}\right)\right]$, clearly $\operatorname{deg}_{H_{i}}(u)=2$ and $b\left(H_{i}\right)=b\left(H_{i} \backslash\{u\}\right)$. Let us prove that $b(H) \neq b(H \backslash\{u\})$ where $H:=\left[V\left(H_{1}\right) \cap V\left(H_{2}\right)\right]=\left[\left\{a_{i^{\prime}}, b_{j^{\prime}}, b_{j_{2}}, \ldots, b_{j_{1}}\right\}\right]$. Indeed, if $j^{\prime}<j_{2}-1$, then the vertices $a_{i^{\prime}}, b_{j^{\prime}}$ joined by an edge form a connected component of $H$, thus $b(H)=2$ and $b(H \backslash\{v\})=3$, and if $j^{\prime}=j_{2}$ or $j^{\prime}=j_{2}-1$, then $b(H)=1$ and $b(H \backslash\{v\})=2$. In both cases this is a contradiction to Lemma 5.1 .

$(\Leftarrow)$ If $G$ is an odd partial band, then $\operatorname{ht}\left(P_{G}\right)=s$. We set $e_{i}:=\left\{a_{j_{i}}, b_{k_{i}}\right\}$ for $1 \leq i \leq s, e_{s+i}:=\left\{a_{i}, a_{i+1}\right\}$ for $1 \leq i<r_{1}$, $e_{s+r_{1}}:=\left\{a_{1}, a_{r_{1}}\right\}, e_{s+r_{1}+i}:=\left\{b_{i}, b_{i+1}\right\}$ for $1 \leq i<r_{2}$ and $e_{s+r_{1}+r_{2}}:=\left\{b_{1}, b_{r_{2}}\right\}$.

For every $i \in\{1, \ldots, s-1\}$, let $w_{i}$ be the even primitive cycle

$$
w_{i}:=\left(b_{k_{i}}, a_{j_{i}}, a_{j_{i}+1}, \ldots, a_{j_{i+1}}, b_{k_{i+1}}, b_{k_{i+1}-1}, \ldots, b_{k_{i}}\right)
$$

and $w_{s}:=\left(b_{k_{s}}, a_{j_{s}}, a_{j_{s}+1}, \ldots, a_{r_{1}}, a_{1}, \ldots, a_{j_{1}}, b_{k_{1}}, \ldots, b_{1}, b_{r_{2}}, \ldots, b_{k_{s}}\right)$.

For every $i \in\{1, \ldots, s\}$ if we write $B_{w_{i}}=x^{\alpha_{i}}-x^{\beta_{i}}$ with $\alpha_{i}, \beta_{i} \in \mathbb{N}^{s+r_{1}+r_{2}}$, then $x_{i} \mid x^{\alpha_{i}}$. Moreover, for every $1 \leq i<s$, $x_{i+1} \mid x^{\alpha_{i}}$ if $j_{i+1}-j_{i}$ is odd and $x_{i+1} \mid x^{\beta_{i}}$ otherwise, and $x_{1} \mid x^{\alpha_{s}}$ if $r_{1}-j_{s}+j_{1}$ is odd and $x_{1} \mid x^{\beta_{s}}$ otherwise.

We denote by $B$ the $s \times\left(s+r_{1}+r_{2}\right)$ matrix whose $i$-th row is $\alpha_{i}-\beta_{i}$. Note that $\Delta_{s}(B)=1$ and for every $j>s$, the $j$-th column of $B$ has only one nonzero entry that can be either +1 or -1 . Hence, by Lemma 2.5 , $B$ is dominating if and only if $B^{\prime}$ is dominating, where $B^{\prime}$ is the $s \times s$ submatrix consisting on the first $s$ columns of $B$. If we denote $B^{\prime}:=\left(b_{i, j}\right)_{1 \leq i, j \leq s}$, then $b_{i, j} \neq 0$ if and only if $j-i \in\{0,1\}$ or $i=s$ and $j=1$; thus if there exists a square submatrix $C$ of $B^{\prime}$ which is mixed, then $C=B^{\prime}$ necessarily. Let us see that $B^{\prime}$ is not mixed; indeed, $r_{1}$ is odd and, if we denote by $l_{1}=j_{2}-j_{1}, l_{2}=j_{3}-j_{2}, \ldots, l_{s-1}=j_{s}-j_{s-1}$ and $l_{s}=r_{1}-j_{s}+j_{1}$; then $r_{1}=l_{1}+\cdots+l_{s}$; thus there exists $1 \leq i \leq s$ such that $l_{i}$ is odd. Then the two nonzero entries in the $i$-th row of $B^{\prime}$ are positive and $B^{\prime}$ is not mixed. Following Theorem 2.4 we can conclude that $P_{G}$ is a complete intersection minimally generated by $\left\{B_{w_{1}}, \ldots, B_{w_{s}}\right\}$.

Now we can state the following structure theorem for complete intersection graphs.

Theorem 6.5. Let $G=[C ; R]$ be a complete intersection connected graph. Then,

- $R$ is a ring graph

- $C$ is either the empty graph, an odd primitive cycle, an odd partial band or $C$ has two connected components which are odd primitive cycles.

Proof. This result is a consequence of the fact that the complete intersection property is hereditary (Theorem 3.4), which allows us to claim that if $G=[C ; R]$ is a complete intersection graph, then both $R$ and $C$ are complete intersection graphs. Thus, by Corollary 3.3 in [11] it follows that $R$ is a ring graph and by Proposition 6.1 and Proposition 6.4 it follows that $C$ is either the empty graph, an odd primitive cycle, an odd partial band or $C$ has two connected components which are odd primitive cycles.

The converse of this statement is not true in general, as the graph in Figure 9 shows. 


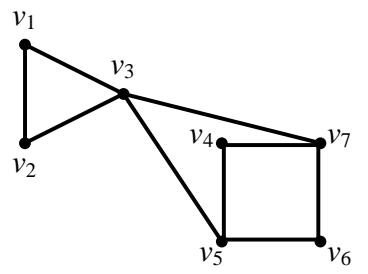

Figure 9: Non complete intersection graph satisfying the conditions in Theorem 6.5

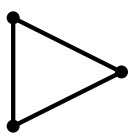

$G_{1}$

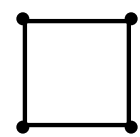

$G_{2}$
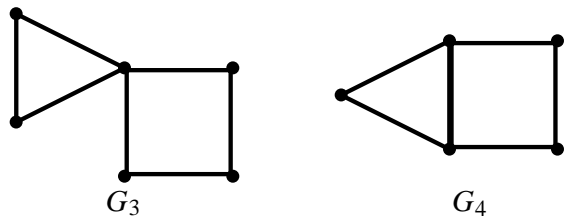

Figure 10: The graph $G_{3}$ is a 1-clique-sum of $G_{1}$ and $G_{2}$, whereas $G_{4}$ is a 2-clique-sum of $G_{1}$ and $G_{2}$

Example 6.6. Let $G=[C ; R]$ be the graph in Figure 9 where $C=\left[\left\{v_{1}, v_{2}, v_{3}\right\}\right]$ is an odd primitive cycle and $R=$ $\left[\left\{v_{4}, v_{5}, v_{6}, v_{7}\right\}\right]$ is a bipartite ring graph. $R$ is a ring graph and $C$ is a complete intersection, nevertheless $G$ is not a complete intersection because it contains $\left[V(R) \cup\left\{v_{3}\right\}\right]$, which is $\mathcal{K}_{2,3}$, as a subgraph; see Corollary 3.9.

Under the hypotheses that $R$ is 2-connected and $C$ is connected, one has the characterization given in Proposition 6.8. In particular, this proposition states that there are either 1 or 2 vertices in $R$ such that every edge connecting $R$ and $C$ is incident to one of these vertices. To state Proposition 6.8 we need a definition.

Definition 6.7. $G$ is a 1-clique-sum of two graphs $G_{1}$ and $G_{2}$ if it is obtained by identifying a vertex $v_{1}$ of $G_{1}$ and a vertex $v_{2}$ of $G_{2}$. Analogously, a 2-clique-sum of $G_{1}$ and $G_{2}$ is obtained by identifying an edge $e_{1}$ of $G_{1}$ and an edge $e_{2}$ of $G_{2}$.

Proposition 6.8. Let $G=[C ; R]$ be a connected graph such that $R$ is 2-connected and $C$ is connected. Then, $G$ is a complete intersection if and only if $R$ is a ring graph and either there exists $u_{1} \in V(R)$ such that $G$ is a 1-clique-sum of $R$ and $\left[V(C) \cup\left\{u_{1}\right\}\right]$, with $\left[V(C) \cup\left\{u_{1}\right\}\right]$ a complete intersection, or there exist two adjacent vertices $u_{1}, u_{2} \in V(R)$ such that $G$ is a 2-clique-sum of $R$ and $\left[V(C) \cup\left\{u_{1}, u_{2}\right\}\right]$, with $\left[V(C) \cup\left\{u_{1}, u_{2}\right\}\right]$ a complete intersection.

This result is an immediate consequence of Lemma 6.9 and Lemma 6.10

Lemma 6.9. Let $G=[C ; R]$ connected graph such that $R$ is 2-connected and $C$ is connected. If $G$ is a complete intersection, then either there exists a vertex $u_{1} \in V(R)$ such that $G$ is a 1 -clique-sum of $R$ and $\left[V(C) \cup\left\{u_{1}\right\}\right]$, or there exist two adjacent vertices $u_{1}, u_{2} \in V(R)$ such that $G$ is a 2-clique-sum of $R$ and $\left[V(C) \cup\left\{u_{1}, u_{2}\right\}\right]$.

Proof. Assume that $G=[C ; R]$ is a complete intersection where $R$ is 2-connected and $C$ is connected. By Theorem 6.5. $C$ is either the empty graph, an odd primitive cycle or an odd partial band and $R$ is a 2-connected ring graph.

Suppose that there exist two edges $e_{1}=\left\{u_{1}, v_{1}\right\}, e_{2}=\left\{u_{2}, v_{2}\right\}$ such that $u_{1} \neq u_{2}, u_{1}, u_{2} \in V(R), v_{1}, v_{2} \in V(C)$ and $u_{1}$ and $u_{2}$ are not adjacent. Let $\mathcal{P}_{1}$ and $\mathcal{P}_{2}$ be two paths in $R$ connecting $u_{1}$ and $u_{2}$ such that $V\left(\mathcal{P}_{1}\right) \cap V\left(\mathcal{P}_{2}\right)=\left\{u_{1}, u_{2}\right\}$ and $\left|V\left(\mathcal{P}_{1}\right) \cup V\left(\mathcal{P}_{2}\right)\right|$ is minimal. Hence, the induced subgraph $\left[V\left(\mathcal{P}_{i}\right)\right]$ is a path graph for $i=1,2$. Since $R$ is bipartite, both $\mathcal{P}_{1}$ and $\mathcal{P}_{2}$ have the same parity. 
First assume that $\mathcal{P}_{1}$ and $\mathcal{P}_{2}$ are even paths. If $v_{1}=v_{2}$, then we set $\mathcal{P}_{3}:=\left(u_{1}, v_{1}, u_{2}\right)$ and $\mathcal{P}_{1}, \mathcal{P}_{2}$ and $\mathcal{P}_{3}$ are all even paths connecting $u_{1}$ and $u_{2}$; but this contradicts Theorem 5.7. If $v_{1} \neq v_{2}$, whenever there exists an even path $\mathcal{P}_{3}^{\prime}$ in $C$ connecting $v_{1}, v_{2}$ we define $\mathcal{P}_{3}:=\left(u_{1}, v_{1}, \mathcal{P}_{3}^{\prime}, v_{2}, u_{2}\right)$, then $\mathcal{P}_{1}, \mathcal{P}_{2}$ and $\mathcal{P}_{3}$ are all even paths connecting $u_{1}$ and $u_{2}$; but this is not possible by Theorem 5.7. It is easy to check that there exists such an even path $\mathcal{P}_{3}^{\prime}$ unless if $C$ is an odd partial band consisting of two odd primitive cycles $C_{1}=\left(a_{1}, \ldots, a_{r_{1}}, a_{1}\right)$ and $C_{2}=\left(b_{1}, \ldots, b_{r_{2}}, b_{2}\right)$ such that $E(C)=E\left(C_{1}\right) \cup E\left(C_{2}\right) \cup\left\{\left\{a_{1}, b_{1}\right\}\right\}$ and $\left\{a_{1}, b_{1}\right\}=\left\{v_{1}, v_{2}\right\}$. In this situation, we set $G^{\prime}:=\left[V(C) \cup V\left(\mathcal{P}_{1}\right) \cup V\left(\mathcal{P}_{2}\right)\right]$ and we have that $G^{\prime}$ is a complete intersection and $\operatorname{deg}_{G^{\prime}}\left(a_{1}\right) \geq 4$, then by Corollary 3.8 there exists a $v \in V\left(G^{\prime}\right)$ of degree 2. If $v \in V(C)$, we can assume that $v \in V\left(C_{1}\right)$ and set $H_{1}:=\left[V\left(C_{1}\right) \cup V\left(\mathcal{P}_{1}\right) \cup\left\{b_{1}\right\}\right]$, then $b\left(H_{1}\right)=b\left(H_{1} \backslash\{v\}\right)=0$ because $\left(u_{1}, \mathcal{P}_{1}, u_{2}, v_{2}, v_{1}, u_{1}\right)$ is an odd cycle in $H_{1} \backslash\{v\}$. Since $b(C)=b(C \backslash\{v\})=0$, it follows by Lemma 5.1 that $b(H)=b(H \backslash\{v\})$ where $H=\left[V(C) \cap V\left(H_{1}\right)\right]=\left[V\left(C_{1}\right) \cup\left\{b_{1}\right\}\right]$. Nevertheless, $b(H)=0$ because $V\left(C_{1}\right) \subset V(H)$ and $b(H \backslash\{v\})=1$ because $H \backslash\{v\}$ is acyclic, so there is a contradiction. If $v \in V\left(\mathcal{P}_{1}\right) \cup V\left(\mathcal{P}_{2}\right)$, we can assume that $v \in V\left(\mathcal{P}_{1}\right)$ and we set $H_{1}:=\left[V\left(\mathcal{P}_{1}\right) \cup V\left(C_{1}\right) \cup\left\{b_{1}\right\}\right]$ and $H_{2}:=\left[V\left(\mathcal{P}_{1}\right) \cup V\left(\mathcal{P}_{2}\right)\right]$, then $b\left(H_{1}\right)=b\left(H_{1} \backslash\{v\}\right)=0$ and $b\left(H_{2}\right)=b\left(H_{2} \backslash\{v\}\right)=1$. Then by Lemma5.1, it follows that $b(H)=b(H \backslash\{v\})$ where $H=\left[V\left(H_{1}\right) \cap V\left(H_{2}\right)\right]=\left[V\left(\mathcal{P}_{1}\right)\right]$. Nevertheless, $b(H)=1$ and $b(H \backslash\{v\})=2$ because $H$ is a path graph and $\operatorname{deg}_{H}(v)=2$, so there is a contradiction.

Assume now that $\mathcal{P}_{1}$ and $\mathcal{P}_{2}$ are odd paths. If $v_{1} \neq v_{2}$, then one can easily find an odd path $\mathcal{P}_{3}^{\prime}$ in $C$ connecting $v_{1}, v_{2}$. Therefore, if we set $\mathcal{P}_{3}:=\left(u_{1}, v_{1}, \mathcal{P}_{3}^{\prime}, v_{2}, u_{2}\right)$, then $\mathcal{P}_{1}, \mathcal{P}_{2}$ and $\mathcal{P}_{3}$ are all odd paths connecting $u_{1}$ and $u_{2}$; but this is not possible by Theorem 5.7. It only suffices to consider the case in which $v_{1}=v_{2}$. Since $v_{1} \in V(C)$, we see that $v_{1}$ belongs to the odd primitive cycle $C^{\prime}=C_{1}$ or $C^{\prime}=C_{2}$ and we set $G^{\prime}:=\left[V\left(C^{\prime}\right) \cup V\left(\mathcal{P}_{1}\right) \cup V\left(\mathcal{P}_{2}\right)\right]$. We claim that every vertex of $C^{\prime}$ different from $v_{1}$ has degree 2. Otherwise there exists $v^{\prime} \in V\left(C^{\prime}\right), v^{\prime} \neq v_{1}$ and $u \in V\left(\mathcal{P}_{1}\right) \cup V\left(\mathcal{P}_{2}\right)$ such that $\left\{u, v^{\prime}\right\} \in E\left(G^{\prime}\right)$, we will assume that $u \in V\left(\mathcal{P}_{1}\right)$. Then, as we proved before, $\left\{u, u_{1}\right\},\left\{u, u_{2}\right\} \in E(R)$. Hence $\left(u_{1}, \mathcal{P}_{2}, u_{2}, u, u_{1}\right)$ is an odd cycle in $R$, but this is not possible because $R$ is bipartite. So we take $v$ any vertex of $C^{\prime}$ different from $v_{1}$ and $H_{i}:=\left[V\left(\mathcal{P}_{i}\right) \cup V\left(C^{\prime}\right)\right]$ for $i=1,2$. Then $C_{i}:=\left(u_{1}, \mathcal{P}_{i}, u_{2}, v_{1}, u_{1}\right)$ is an odd cycle with $v \notin V\left(C_{i}\right) \subset V\left(H_{i}\right)$, which gives $b\left(H_{i}\right)=b\left(H_{i} \backslash\{v\}\right)=0$. Then by Lemma5.1, it follows that $b(H)=b(H \backslash\{v\})$ where $H=\left[V\left(H_{1}\right) \cap V\left(H_{2}\right)\right]=\left[V\left(C^{\prime}\right) \cup\left\{u_{1}, u_{2}\right\}\right]$. Nevertheless, since $H \backslash\{v\}$ is acyclic and $V(C) \subset V(H)$, we have that $b(H)=0$ and $b(H \backslash\{v\})=1$, a contradiction.

To sum up, we have proved that whenever $\left\{u_{1}, v_{1}\right\},\left\{u_{2}, v_{2}\right\} \in E(G)$ with $v_{1}, v_{2} \in V(C), u_{1}, u_{2} \in V(R)$ and $u_{1} \neq u_{2}$, then $\left\{u_{1}, u_{2}\right\} \in E(G)$. If there exist three different vertices $u_{1}, u_{2}, u_{3} \in E(G)$ such that $\left\{u_{i}, v_{i}\right\} \in E\left(G^{\prime}\right)$ for some $v_{1}, v_{2}, v_{3} \in V(C)$, then $u_{1}, u_{2}, u_{3}$ form a triangle in $R$, but this is not possible because $R$ is bipartite, and the lemma follows.

Lemma 6.10. Let $G$ be a 1-clique-sum or a 2-clique-sum of a graph $H$ and a bipartite ring graph $R$. Then, $G$ is a complete intersection $\Longleftrightarrow H$ is a complete intersection.

Proof. One implication is obvious because $H$ is an induced subgraph of $G$. Since bipartite ring graphs are constructed by performing 1-clique-sums and 2-clique-sums of even primitive cycles and edges, we only have to prove that $G$ is a complete intersection when it is a 1-clique-sum or a 2-clique-sum of a complete intersection graph $H$ and $K$, where $K$ is either an even primitive cycle or an edge. If $K$ is an edge $e=\left\{v_{1}, v_{2}\right\}$ and $G$ is a 1-clique-sum of $H$ and $K$, then either $\operatorname{deg}_{G}\left(v_{1}\right)=1$ or $\operatorname{deg}_{G}\left(v_{2}\right)=1$ and, by Theorem 4.8, $G$ is a complete intersection. So assume that $K$ is an even primitive cycle $C$. Let $\mathfrak{B}=\left\{B_{w_{1}}, \ldots, B_{w_{r}}\right\}$ a minimal set of generators of $P_{H}$ where $r=\operatorname{ht}\left(P_{H}\right)$ and consider $\mathfrak{B}^{\prime}:=\mathfrak{B} \cup\left\{B_{C}\right\}$. If we prove that $\mathfrak{B}^{\prime}$ generates $P_{G}$, then $G$ is a complete intersection because $\operatorname{ht}\left(P_{G}\right)=\operatorname{ht}\left(P_{H}\right)+1$. We write $B_{w_{i}}:=x^{\alpha_{i}}-x^{\beta_{i}}$ for $i=1, \ldots, r$ and call $B$ the matrix whose $i$-th row is $\gamma_{i}:=\alpha_{i}-\beta_{i}$, then $B$ is dominating and $\Delta_{r}(B)=1$. We also write $B_{C}:=x^{\alpha}-x^{\beta}$ and $B^{\prime}$ the matrix obtained by adding a new row $\gamma:=\alpha-\beta$ to $B$, let us see that $B^{\prime}$ is dominating and $\Delta_{r+1}\left(B^{\prime}\right)=1$. Indeed, $C$ is a cycle which involves at most one edge of $H$, then by Lemma $2.5 B^{\prime}$ is also dominating and $\gamma$ has only +1 and -1 in the entries corresponding to edges in $E(C)$, then $\Delta_{r+1}\left(B^{\prime}\right)=\Delta_{r}(B)=1$, which proves the lemma.

Next we deal with the problems of characterizing when $\left[V(C) \cup\left\{u_{1}\right\}\right]$ is a complete intersection, with $u_{1} \in V(R)$, and when $\left[V(C) \cup\left\{u_{1}, u_{2}\right\}\right]$ is a complete intersection, where $u_{1}, u_{2} \in V(R)$ are adjacent vertices. By Theorem 3.4 , when either $\left[V(C) \cup\left\{u_{1}\right\}\right]$ or $\left[V(C) \cup\left\{u_{1}, u_{2}\right\}\right]$ is a complete intersection, one has that so is $C$ and then, by Theorem 6.5, $C$ is either an odd primitive cycle or an odd partial band because $C$ is connected. Thus, we will study the complete 

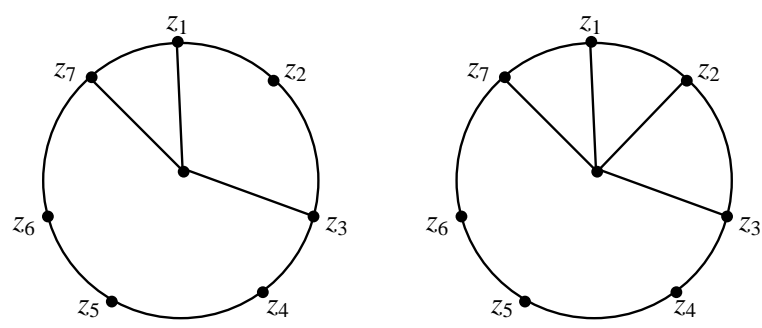

Figure 11: Two CI-odd-partial-wheels

intersection property on the following graphs:

1. $\left[V(C) \cup\left\{u_{1}\right\}\right]$, where $C$ is an odd primitive cycle,

2. $\left[V(C) \cup\left\{u_{1}, u_{2}\right\}\right]$, where $u_{1}, u_{2}$ are adjacent vertices and $C$ is an odd primitive cycle,

3. $\left[V(C) \cup\left\{u_{1}\right\}\right]$, where $C$ is an odd partial band, and

4. $\left[V(C) \cup\left\{u_{1}, u_{2}\right\}\right]$, where $u_{1}, u_{2}$ are adjacent vertices and $C$ is an odd partial band.

The following four lemmas study all these situations. Let us start with one definition.

Definition 6.11. An odd partial wheel $W$ consists of an odd primitive cycle $C$, a vertex $x \notin V(C)$ and at least one edge connecting $x$ and $C$. The vertex $x$ is called the central vertex of $W$, and $C$ is called the principal cycle of $W$.

Moreover $W$ is a CI-odd-partial-wheel if $C=\left(z_{1}, \ldots, z_{r}, z_{1}\right)$ and $N_{W}(x)=\left\{z_{1}, z_{s_{2}}, \ldots, z_{s_{k}}\right\}$, where $k \geq 1,1<s_{2}<$ $\cdots<s_{k-1}<s_{k}, s_{3}, \ldots, s_{k}$ are odd and either $s_{2}=2$ or $s_{2}$ is odd (see Figure 11).

Lemma 6.12. Let $W$ be an odd partial wheel. $W$ is a complete intersection if and only if $W$ is a CI-odd-partial-wheel.

Proof. Let $x$ be the central vertex and $C$ the principal cycle of $W$, we denote $r:=|V(C)|$.

$(\Rightarrow)$ We proceed by induction on $r$, if $r=3$ then $W$ is always a CI-odd-partial-wheel. If $r \geq 5 \operatorname{and} \operatorname{deg}(x) \leq 2$ then evidently $W$ is a CI-odd-partial-wheel. So we can assume that $\operatorname{deg}_{W}(x) \geq 3$, then there exists $v \in V(C)$ such that $\operatorname{deg}_{W}(v)=2$, otherwise $\operatorname{deg}_{W}(x)=r>3$ and this contradicts Corollary 3.8. Thus we consider $W^{\prime}:=W_{v}^{c}$, which is a complete intersection odd partial wheel and, by induction hypothesis, it is a CI-odd-partial-wheel. Hence $W^{\prime}$ has a central vertex $x$ and a principal cycle $C^{\prime}=\left(z_{1}^{\prime}, \ldots, z_{r-2}^{\prime}\right)$ such that $N_{W^{\prime}}(x)=\left\{z_{1}^{\prime}, z_{t_{1}}^{\prime}, \ldots, z_{t_{k}}^{\prime}\right\}$ where $1<t_{1}<\cdots t_{k} \leq r-2$, with $t_{2}, \ldots, t_{k}$ odd and $t_{1}=2$ or $t_{1}$ is odd. Thus $C=\left(z_{1}, \ldots, z_{r}, z_{1}\right)$ and there exists $l \in\{2, \ldots, r-1\}$ such that

$$
\left\{x, z_{i}^{\prime}\right\} \in E\left(W^{\prime}\right) \Longleftrightarrow\left\{\begin{array}{ccc}
\left\{x, z_{i}\right\} \in E(W) & \text { and } & i \leq l-1,
\end{array}\right. \text { or }
$$

If $\left\{x, z_{1}\right\} \notin E(W)$ then we set $y_{i}:=z_{i+2}$ for $1 \leq i \leq r-2, y_{1}=z_{r-1}$ and $y_{2}=z_{r}$ and have that $W$ is a CI-odd-partialwheel. If $l \neq 3$ or $\left\{x, z_{4}\right\} \notin E(W)$, then $W$ also is a CI-odd-partial-wheel. So it remains to study when $\left\{x, z_{1}\right\},\left\{x, z_{4}\right\}$ and $l=3$, we firstly assume that $\left\{x, z_{2}\right\} \in E(W)$. If $\operatorname{deg}_{W}(x)=3$, then we set $y_{i}:=z_{i+1}$ for $1 \leq i<r$ and $y_{r}:=z_{1}$ and have that $C=\left(y_{1}, \ldots, y_{r}, y_{1}\right)$ with $N_{W}(x)=\left\{y_{1}, y_{3}, y_{r}\right\}$; thus it is a CI-odd-partial-wheel. If $N_{W}(x)=\left\{z_{1}, z_{2}, z_{4}, z_{r}\right\}$, then setting $y_{1}=z_{r}, y_{i}=z_{i-1}$ for $2 \leq i \leq r$ we have that $N_{W}(x)=\left\{y_{1}, y_{2}, y_{3}, y_{5}\right\}$ and $W$ is a CI-odd-partial-wheel. If $\operatorname{deg}_{W}(x) \geq 4$ and $N_{W}(x) \neq\left\{z_{1}, z_{2}, z_{4}, z_{r}\right\}$, then we take $j=\min \left\{i>4 \mid z_{i} \in N_{W}(x)\right\}$ and there are two odd cycles $C_{1}:=\left(z_{1}, z_{2}, x, z_{1}\right)$ and $C_{2}:=\left(z_{4}, \ldots, z_{j}, x, z_{4}\right)$ because $j$ is odd, but there is no edge connecting $C_{1}$ and $C_{2}$ and this is impossible by Lemma 6.2

Now we assume that $l=3$ and $\left\{x, z_{2}\right\} \notin E(W)$. If $\operatorname{deg}_{W}(x)=3$ and $\left\{x, z_{j}\right\} \in E(W)$ for $j=5$ or $j=r$, then $W$ is a CI-odd-partial-wheel. Indeed, if $j=r$ we set $y_{1}:=z_{r}, y_{i}=z_{i-1}$ for $2 \leq i \leq r$, then $N_{W}(x)=\left\{y_{1}, y_{2}, y_{5}\right\}$ and $W$ is a CI-odd-partial-wheel. If $j=5$ one can proceed analogously. 


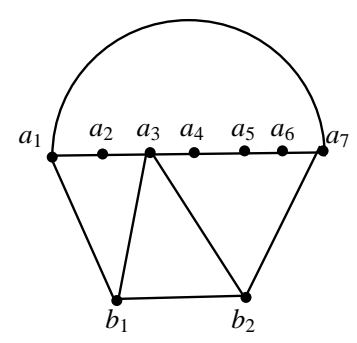

Figure 12: A CI-double-wheel

If $\operatorname{deg}_{W}(x) \geq 3$ and we are not in the previous situations, then $\operatorname{deg}_{G}\left(z_{2}\right)=2$ and there exist $5 \leq j_{1} \leq j_{2} \leq r$ such that $z_{j_{1}}, z_{j_{2}} \in N_{W}(x),\left(j_{1}, j_{2}\right) \neq(5,5)$ and $\left(j_{1}, j_{2}\right) \neq(r, r)$. Since $j_{1}$ and $j_{2}$ are odd, we consider the two even cycles $C_{1}:=\left(z_{1}, \ldots, z_{j_{1}}, x, z_{1}\right)$ and $C_{2}:=\left(z_{j_{2}}, \ldots, z_{r}, z_{1}, \ldots, z_{4}, x, z_{j_{2}}\right)$. Set $H_{i}:=\left[V\left(C_{i}\right)\right]$ for $i=1,2$, then $b\left(H_{i}\right)=b\left(H_{i} \backslash\left\{z_{2}\right\}\right)$, however if one takes $H:=\left[V\left(H_{1}\right) \cap V\left(H_{2}\right)\right]$, then $b(H)=0$ and $b\left(H \backslash\left\{z_{2}\right\}\right)=1$, which contradicts Lemma 5.1]

$(\Leftarrow)$ We write $C=\left(z_{1}, \ldots, z_{r}, z_{1}\right)$ and $N_{W}(x)=\left\{z_{1}, z_{s_{2}}, \ldots, z_{s_{k}}\right\}$ where $s_{1}:=1<s_{2}<\cdots<s_{k}, s_{3}, \ldots, s_{k}$ are odd and either $s_{2}=2$ or $s_{2}$ is odd. If $s_{2}$ is odd, we set $\bar{W}$ the odd partial wheel with principal cycle $C^{\prime}=\left(z_{1}^{\prime}, \ldots, z_{r+2}^{\prime}, z_{1}^{\prime}\right)$, central vertex $x^{\prime}$ and $\left\{x^{\prime}, z_{i}^{\prime}\right\} \in E(\bar{W})$ if and only if $\left\{x, z_{i}\right\} \in E(W)$. Clearly $\operatorname{deg}_{\bar{W}}\left(z_{r+1}^{\prime}\right)=\operatorname{deg}_{\bar{W}}\left(z_{r+2}^{\prime}\right)=2$ and $W=\bar{W}_{z_{r+1}^{\prime}}^{c}$, so if we prove that $\bar{W}$ is a complete intersection, then by Proposition 5.3 so is $W$. We set $R:=\bar{W} \backslash\left\{z_{r+2}^{\prime}\right\}$ and have that $b(\bar{W})=0$ and $b(R)=1$, then by Theorem $4.8, \bar{W}$ is a complete intersection if and only if so is $R$. Since $R$ is a bipartite ring graph, we conclude that $R, \bar{W}$ and $W$ are complete intersections.

Suppose now that $s_{2}=2$, we denote $e_{i}=\left\{x, z_{s_{i}}\right\}$ for $1 \leq i \leq k, e_{k+i}=\left\{z_{i}, z_{i+1}\right\}$ and $e_{k+r}=\left\{z_{1}, z_{r}\right\}$. W has $r+1$ vertices and $r+k$ edges; thus ht $\left(P_{W}\right)=k-1$. Consider the even cycles $C_{1}:=\left(x, z_{s_{k}}, z_{s_{k}+1}, \ldots, z_{r}, z_{1}, z_{2}, x\right)$, $C_{2}:=\left(x, z_{1}, z_{2}, \ldots, z_{s_{3}}, x\right)$ and $C_{i}:=\left(x, z_{s_{i}}, z_{s_{i}+1}, \ldots, z_{s_{i+1}}, x\right)$ for $3 \leq i \leq k-1$; then

$$
\begin{gathered}
B_{C_{1}}=x_{k} x_{k+1} x_{k+s_{k}+1} \cdots x_{k+r-1}-x_{2} x_{k+s_{k}} \cdots x_{k+r}, \\
B_{C_{2}}=x_{1} x_{k+2} \cdots x_{k+s_{3}-1}-x_{3} x_{k+1} \cdots x_{k+s_{3}-2}, \text { and } \\
B_{C_{i}}=x_{i} x_{k+s_{i}+1} \cdots x_{k+s_{i+1}-1}-x_{i+1} x_{k+s_{i}} \cdots x_{k+s_{i+1}-2} \text { for } 3 \leq i \leq k-1,
\end{gathered}
$$

let us prove that $P_{W}=\left(B_{C_{1}}, \ldots, B_{C_{k-1}}\right)$. We set $B_{C_{i}}=x^{\alpha_{i}}-x^{\beta_{i}}$ and $\gamma_{i}:=\alpha_{i}-\beta_{i}$ for $1 \leq i \leq k-1$; then,

$$
\begin{gathered}
\gamma_{1}:=-e_{2}+e_{k}+e_{k+1}-e_{k+s_{k}}+e_{k+s_{k}+1}-\cdots+e_{k+r-1}-e_{k+r} \in \mathbb{Z}^{k+r}, \\
\gamma_{2}:=e_{1}-e_{3}-e_{k+1}+e_{k+2}-\cdots-e_{k+s_{3}-2}+e_{k+s_{3}-1} \in \mathbb{Z}^{k+r}, \text { and } \\
\gamma_{i}:=e_{i}-e_{i+1}-e_{k+s_{i}}+e_{k+s_{i}+1}-e_{k+s_{i}+2}+\cdots-e_{k+s_{i+1}-2}+e_{k+s_{i+1}-1} \in \mathbb{Z}^{k+r}
\end{gathered}
$$

for $3 \leq i \leq k-1$, and denote by $B$ the $(k-1) \times(k+r)$ matrix whose $i$-th row is $\gamma_{i}$. It is evident that $\Delta_{k-1}(B)=1$ and for every $j \in\{1,2, k+2, \ldots, k+r\}$ the $j$-th column of $B$ has only one nonzero entry; thus by Lemma 2.5 $B$ is dominating if and only if $B^{\prime}$ is dominating where $B^{\prime}$ is the $k-1 \times k-1$ matrix consisting of the columns $3,4, \ldots, k+1$ of $B$. $B^{\prime}$ has exactly two nonzero entries in each row and in each column and both nonzero entries in the first row of $B^{\prime}$ are positive. Hence, $B^{\prime}$ is dominating. Therefore we conclude that $W$ is a complete intersection and $P_{W}=\left(B_{C_{1}}, \ldots, B_{C_{k-1}}\right)$.

Definition 6.13. A connected graph $G$ is called a CI-double-wheel if its vertex set is $V(G)=V(C) \cup\left\{b_{1}, b_{2}\right\}$, where $C=\left(a_{1}, \ldots, a_{r}, a_{1}\right)$ is an odd primitive cycle and $E(G)=E(C) \cup\left\{\left\{b_{1}, b_{2}\right\},\left\{b_{1}, a_{j_{1}}\right\}, \ldots,\left\{b_{1}, a_{j_{s}}\right\},\left\{b_{2}, a_{k_{1}}\right\}, \ldots,\left\{b_{2}, a_{k_{t}}\right\}\right\}$, for some $s, t \geq 1,1 \leq j_{1}<\cdots<j_{s} \leq k_{1}<\cdots<k_{t} \leq r$ and $j_{1}, \ldots, j_{s}, k_{1}, \ldots, k_{t}$ are odd (see Figure 12).

Lemma 6.14. Let $G$ be a connected graph with $V(G)=V(C) \cup\left\{b_{1}, b_{2}\right\}$ where $C$ is an odd primitive cycle, $\left\{b_{1}, b_{2}\right\} \in$ $E(G)$ and $\operatorname{deg}_{G}\left(b_{1}\right), \operatorname{deg}_{G}\left(b_{2}\right) \geq 2$. Then, $G$ is a complete intersection if and only if $G$ is a CI-double-wheel. 
Proof. $(\Rightarrow)$ We proceed by induction on $r:=|V(C)|$. If $r=3$, then by Corollary 3.7 it has $\leq 7$ edges. If every vertex of $C$ has degree $\geq 3$, then $G$ has a subgraph $\mathcal{K}_{2,3}$, which contradicts Corollary 3.9 Thus one can write $V(G)=V(C) \cup$ $\left\{b_{1}, b_{2}\right\}$, where $C=\left(a_{1}, a_{2}, a_{3}, a_{1}\right), \operatorname{deg}_{G}\left(a_{2}\right)=\operatorname{deg}_{G}\left(b_{2}\right)=2$ and $\operatorname{deg}_{G}\left(b_{1}\right) \leq 3$, thus $G$ is a CI-double wheel. Assume now that $r \geq 5$, we claim that there exists a vertex of degree 2 in $C$. Indeed, if $\operatorname{deg}_{G}(v) \geq 3$ for every $v \in V(C)$, then we have that $4 r+2 \leq 2|E(G)| \leq 3|V(G)|=3 r+6$, which contradicts Corollary 3.7. Therefore we take $v \in V(C)$ of degree 2 and consider $G^{\prime}:=G_{v}^{c}$, which is a CI-double wheel by induction hypothesis. Thus $V\left(G^{\prime}\right)=V\left(C^{\prime}\right) \cup\left\{b_{1}, b_{2}\right\}$, where $C^{\prime}=$ $\left(a_{1}^{\prime}, \ldots, a_{r-2}^{\prime}, a_{1}^{\prime}\right)$ is an odd primitive cycle and $E\left(G^{\prime}\right)=E\left(C^{\prime}\right) \cup\left\{\left\{b_{1}, b_{2}\right\},\left\{b_{1}, a_{j_{1}}^{\prime}\right\}, \ldots,\left\{b_{1}, a_{j_{s}}^{\prime}\right\},\left\{b_{2}, a_{k_{1}}^{\prime}\right\}, \ldots,\left\{b_{2}, a_{k_{t}}^{\prime}\right\}\right\}$ with $1 \leq j_{1}<\cdots<j_{s} \leq k_{1}<\cdots<k_{t} \leq r$ and $j_{1}, \ldots, j_{s}, k_{1}, \ldots, k_{t}$ are odd. Moreover, there exists $l \in\{2, \ldots, r-1\}$ such that

$$
\left\{b_{i}, a_{j}^{\prime}\right\} \in E\left(G^{\prime}\right) \Longleftrightarrow\left\{\begin{array}{ccc}
\left\{b_{i}, a_{j}\right\} \in E(G) & \text { and } & j \leq l-1, \\
\left\{b_{i}, a_{j+2}\right\} \in E(G) & \text { and } & j \geq l-1
\end{array}\right. \text { or }
$$

If $\left\{b_{1}, a_{l+1}\right\} \notin E(G),\left\{b_{2}, a_{l-1}\right\} \notin E(G) \operatorname{or} \operatorname{deg}\left(b_{1}\right)=\operatorname{deg}\left(b_{2}\right)=2$, then $G$ is a CI-double wheel. Therefore if $G$ is not a CI-double-wheel one can assume that $\left\{b_{1}, a_{l+1}\right\},\left\{b_{2}, a_{l-1}\right\} \in E(G)$ and $\operatorname{deg}\left(b_{1}\right)>2$. Thus, $l-1=j_{s}=k_{1}, \ldots, k_{t}$ are odd and $\left\{b_{1}, a_{j_{1}}\right\},\left\{b_{1}, a_{l+1}\right\},\left\{b_{2}, a_{l-1}\right\} \in E(G)$.

We separate two cases, if $b_{2}$ has degree $>2$ then $\left\{b_{2}, a_{k_{t}}^{\prime}\right\} \in E\left(G^{\prime}\right)$ and $\left\{b_{2}, a_{k_{t}+2}\right\} \in E(G)$, then there exist three even paths

$$
\begin{gathered}
\mathcal{P}_{1}:=\left(a_{l-1}, a_{l}, a_{l+1}\right), \mathcal{P}_{2}:=\left(a_{l-1}, a_{l-2}, \ldots, a_{j_{1}}, b_{1}, a_{l+1}\right) \text { and } \\
\mathcal{P}_{3}:=\left(a_{l-1}, b_{2}, a_{k_{t}+2}, a_{k_{t}+1}, \ldots, a_{l+1}\right)
\end{gathered}
$$

connecting $a_{l-1}$ and $a_{l+1}$ and $V\left(\mathcal{P}_{i}\right) \cap V\left(\mathcal{P}_{j}\right)=\left\{a_{l-1}, a_{l+1}\right\}$ for $1 \leq i<j \leq 3$, which is not possible by Theorem 5.7 If $b_{2}$ has degree 2 and $j_{1}=l-1$, then $G$ is a CI-double-wheel. Hence we assume that $b_{2}$ has degree 2 and $j_{1}<l-1$ and we consider the even cycles $C_{1}:=\left(b_{1}, b_{2}, a_{l-1}, a_{l}, \ldots, a_{r}, a_{1}, \ldots, a_{j_{1}}, b_{1}\right)$ and $C_{2}:=$ $\left(b_{1}, b_{2}, a_{l-1}, a_{l-2}, \ldots, a_{1}, a_{r}, \ldots, a_{l+1}, b_{1}\right)$, and set $H_{i}:=\left[V\left(C_{i}\right)\right]$, then $b\left(H_{i}\right)=b\left(H_{i} \backslash\left\{b_{2}\right\}\right)=1$. However setting $H:=\left[V\left(C_{1}\right) \cap V\left(C_{2}\right)\right]=\left[\left\{a_{1}, \ldots, a_{j_{1}}, a_{l-1}, a_{l+1}, \ldots, a_{r}, b_{1}, b_{2}\right\}\right]$, if $\left\{b_{1}, a_{l-1}\right\} \notin E(G)$, then $b(H)=0$ because $H$ is connected and the odd cycle $C_{3}:=\left(b_{1}, a_{l+1}, \ldots, a_{r}, a_{1}, \ldots, a_{j_{1}}, b_{1}\right)$ is in $H$, and $b\left(H \backslash\left\{b_{2}\right\}\right)=1$ because $a_{l-1}$ is an isolated vertex in $H \backslash\left\{b_{2}\right\}$. It only remains to consider the situation in which $\left\{b_{1}, a_{l-1}\right\} \in E(G)$; in this case $G$ is a 2-connected graph, there are two odd cycles $C_{3}=\left(b_{1}, a_{l-1}, b_{2}, b_{1}\right)$ and $C_{4}=\left(b_{1}, a_{l+1}, \ldots, a_{r}, a_{1}, \ldots, a_{j_{1}}, b_{1}\right)$ with a vertex in common and there is no edge connecting them, a contradiction to Lemma 6.2

$(\Leftarrow)$ Denote by $G^{\prime}$ the graph obtained by adding a new vertex $b_{3}$ and two edges $\left\{b_{1}, b_{3}\right\}$ and $\left\{b_{2}, b_{3}\right\}$, then $G^{\prime}$ is an odd partial band and by Proposition $6.4 G^{\prime}$ is a complete intersection. Furthermore, $G=G^{\prime} \backslash\left\{b_{3}\right\}$, then $G$ is a complete intersection.

Definition 6.15. A CI-vertex-band is a graph $G$ with vertices $V\left(C_{1}\right) \cup V\left(C_{2}\right) \cup\{c\}$, where $C_{1}=\left(a_{1}, \ldots, a_{r}, a_{1}\right)$ and $C_{2}=\left(b_{1}, \ldots, b_{s}, b_{1}\right)$ are vertex disjoint odd primitive cycles and

$$
E(G)=E\left(C_{1}\right) \cup E\left(C_{2}\right) \cup\left\{\left\{a_{1}, b_{1}\right\},\left\{a_{1}, b_{i_{2}}\right\} \ldots\left\{a_{1}, b_{i_{k}}\right\},\left\{c, a_{2}\right\},\left\{c, a_{r}\right\}\right\},
$$

for some $k \geq 1, i_{2}<\cdots<i_{k} \leq s$ and $i_{2}, \ldots, i_{k}$ are odd (see Figure 13).

Lemma 6.16. Let $G$ be a connected graph with $V(G)=V(C) \cup\{c\}$ where $C$ is an odd partial band. Then, $G$ is a complete intersection if and only if $\operatorname{deg}_{G}(c)=1$ or $G$ is a CI-vertex-band.

Proof. $(\Rightarrow)$ We denote by $C_{1}$ and $C_{2}$ the two vertex disjoint odd primitive cycles such that $V(C)=V\left(C_{1}\right) \cup V\left(C_{2}\right)$. We first aim to prove that $c$ has degree $\leq 2$. For this purpose we will prove that if $v \in V(C)$ has degree 2 and does not belong to a triangle, then $\operatorname{deg}_{G}(c)=\operatorname{deg}_{G_{v}^{c}}(c)$. Suppose that $\operatorname{deg}_{G}(c)>\operatorname{deg}_{G_{v}^{c}}(c)$, this means that $N_{G}(v)=\left\{v_{1}, v_{2}\right\}$ and $\left\{v_{1}, c\right\},\left\{v_{2}, c\right\} \in E(G)$. Then we consider $H:=\left[\left\{v, v_{1}, v_{2}, c\right\}\right]$ and have that $v \in V(H) \cap V(C), b(H)=b(H \backslash\{v\})=1$, $b(C)=b(C \backslash\{v\})=0$. However, considering $H^{\prime}:=[V(H) \cap V(C)]=\left[\left\{v, v_{1}, v_{2}\right\}\right]$, then $b\left(H^{\prime} \backslash\{v\}\right)=b\left(H^{\prime}\right)+1$, which contradicts Lemma 5.1 


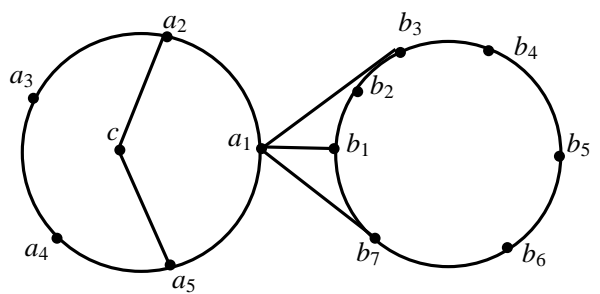

Figure 13: A CI-vertex-band

Suppose that $\operatorname{deg}_{G}(c) \geq 3$. For every $v \in V(C)$ of degree 2 which does not belong to a triangle we consider $G_{c}^{v}$ and we repeat this until we get a graph $G^{\prime}$ in which every vertex of degree 2 belongs to a triangle, then we have proved that $\operatorname{deg}_{G}(c)=\operatorname{deg}_{G^{\prime}}(c)$. Note that $V\left(G^{\prime}\right)=V\left(C^{\prime}\right) \cup\{c\}$ where $C^{\prime}$ is an odd partial wheel with primitive cycles $C_{1}^{\prime}$ and $C_{2}^{\prime}$. Since $G^{\prime}$ has an odd number of vertices, we have that it cannot be a band or a Möbius band and there exists a $v \in V\left(G^{\prime}\right)$ of degree 2, say $v \in V\left(C_{1}^{\prime}\right)$. By construction $C_{1}^{\prime}$ has to be a triangle, let us prove that $C_{2}^{\prime}$ is also a triangle. Suppose that $C_{2}^{\prime}$ is not a triangle, then $s:=\left|V\left(C_{2}^{\prime}\right)\right| \geq 5$ and $\operatorname{deg}_{G^{\prime}}(u) \geq 3$ for every $u \in V\left(C_{2}^{\prime}\right)$, which implies that $\left|E\left(G^{\prime}\right)\right| \geq 2 s+3$ and $\left|V\left(G^{\prime}\right)\right|=s+4$ and by Corollary 3.7 we have that $2\left|E\left(G^{\prime}\right)\right| \leq 3\left|V\left(G^{\prime}\right)\right|$; thus $s=5$ and $\left|E\left(G^{\prime}\right)\right|=13$. This means that $\operatorname{deg}_{G^{\prime}}(z)=3$ for every $z \in V\left(C_{2}^{\prime}\right)$ and $\{c, u\} \notin E\left(G^{\prime}\right)$ for every $u \in V\left(C_{1}^{\prime}\right)$. By symmetry, one can assume that $C_{1}^{\prime}=\left(a_{1}, a_{2}, a_{3}\right), C_{2}^{\prime}=\left(b_{1}, b_{2}, b_{3}, b_{4}, b_{5}, b_{1}\right)$ and $E\left(G^{\prime}\right)=E\left(C_{1}^{\prime}\right) \cup E\left(C_{2}^{\prime}\right) \cup\left\{\left\{a_{1}, b_{1}\right\}\right\} \cup E$, where $E$ is one of these:

(a) $E=\left\{\left\{b_{2}, c\right\},\left\{b_{3}, c\right\},\left\{b_{4}, c\right\},\left\{b_{5}, c\right\}\right\}$,

(b) $E=\left\{\left\{b_{2}, a_{1}\right\},\left\{b_{3}, c\right\},\left\{b_{4}, c\right\},\left\{b_{5}, c\right\}\right\}$,

(c) $E=\left\{\left\{b_{2}, a_{2}\right\},\left\{b_{3}, c\right\},\left\{b_{4}, c\right\},\left\{b_{5}, c\right\}\right\}$,

(d) $E=\left\{\left\{b_{2}, c\right\},\left\{b_{3}, a_{1}\right\},\left\{b_{4}, c\right\},\left\{b_{5}, c\right\}\right\}$, or

(e) $E=\left\{\left\{b_{2}, c\right\},\left\{b_{3}, a_{2}\right\},\left\{b_{4}, c\right\},\left\{b_{5}, c\right\}\right\}$.

If (a) occurs we set $H_{i}:=\left[V\left(C_{1}^{\prime}\right) \cup\left\{c, b_{1}, b_{i-1}, b_{i}\right\}\right]$ and have that $b\left(H_{i}\right)=b\left(H_{i} \backslash\left\{a_{2}\right\}\right)=0$ for $i=3,5$; however if one sets $H:=\left[V\left(H_{3}\right) \cap V\left(H_{5}\right)\right]=\left[V\left(C_{1}^{\prime}\right) \cup\left\{c, b_{1}\right\}\right]$, then $b(H)=1$ and $b\left(H \backslash\left\{a_{2}\right\}\right)=2$, which is impossible by Lemma 5.1. If (b) or (c) holds, we set $\mathcal{P}_{1}:=\left(b_{3}, b_{4}, b_{5}\right), \mathcal{P}_{2}:=\left(b_{3}, c, b_{5}\right)$, in (b) we also set $\mathcal{P}_{3}:=\left(b_{3}, b_{2}, a_{1}, b_{1}, b_{5}\right)$ and in (c) we also set $\mathcal{P}_{3}:=\left(b_{3}, b_{2}, a_{2}, a_{3}, a_{1}, b_{1}, b_{5}\right)$; in both situations we have three even paths connecting $b_{3}$ and $b_{5}$, but this is not possible by Theorem 5.7 In the last two cases we set $\mathcal{P}_{1}:=\left(b_{3}, b_{2}, b_{1}\right), \mathcal{P}_{2}:=\left(b_{3}, b_{4}, c, b_{5}, b_{1}\right)$, in (d) we also set $\mathcal{P}_{3}:=\left(b_{3}, a_{1}, b_{1}\right)$ and in (e) we also set $\mathcal{P}_{3}:=\left(b_{3}, a_{2}, a_{3}, a_{1}, b_{1}\right)$; but this is not possible again by Theorem 5.7

So assume now that both $C_{1}^{\prime}$ and $C_{2}^{\prime}$ are triangles and $\operatorname{deg}_{G^{\prime}}(c) \geq 3$, then necessarily $\operatorname{deg}_{G^{\prime}}(c)=3$ and $E\left(C^{\prime}\right)=$ $E\left(C_{1}^{\prime}\right) \cup E\left(C_{2}^{\prime}\right) \cup\left\{\left\{a_{1}, b_{1}\right\}\right\}$, otherwise $2\left|E\left(G^{\prime}\right)\right|>3\left|V\left(G^{\prime}\right)\right|$. Then one can write $C_{1}^{\prime}=\left(a_{1}, a_{2}, a_{3}, a_{1}\right), C_{2}^{\prime}=\left(b_{1}, b_{2}, b_{3}, b_{1}\right)$ and $N_{G^{\prime}}(c)$ is one of these:

- $N_{G^{\prime}}(c)=\left\{b_{1}, b_{2}, b_{3}\right\}$,

- $N_{G^{\prime}}(c)=\left\{a_{1}, b_{1}, b_{2}\right\}$,

- $N_{G^{\prime}}(c)=\left\{a_{2}, b_{1}, b_{2}\right\}$,

- $N_{G^{\prime}}(c)=\left\{a_{1}, b_{2}, b_{3}\right\}$, or

- $N_{G^{\prime}}(c)=\left\{a_{2}, b_{2}, b_{3}\right\}$.

We set $u:=a_{3}, H_{1}:=C^{\prime}$ and $H_{2}:=G^{\prime} \backslash\left\{b_{3}\right\}$ in the first three cases and $H_{2}:=G^{\prime} \backslash\left\{b_{1}\right\}$ in the last two. In all of them $b\left(H_{1}\right)=b\left(H_{1} \backslash\{u\}\right)=0, b\left(H_{2}\right)=b\left(H_{2} \backslash\{u\}\right)=0$. However, $b(H) \neq b(H \backslash\{u\})$ where $H:=\left[V\left(H_{1}\right) \cap V\left(H_{2}\right)\right]$, a contradiction to Lemma5.1 Thus $\operatorname{deg}_{G}(c) \leq 2$. 
If $\operatorname{deg}_{G}(c)=2$, since $b(G)=b(G \backslash\{c\})=0$, by Theorem 4.8 we get that $P_{G}=P_{C} \cdot k\left[x_{1}, \ldots, x_{n}\right]+\left(B_{w}\right)$, where $w$ is an even closed walk with

$$
V(w)=\{c\} \cup N_{G}(c) \cup\{v \in V(G) \mid b(G \backslash\{v\})<b(C \backslash\{v\})\} .
$$

We assume that $C_{1}=\left(a_{1}, \ldots, a_{r}, a_{1}\right)$ and $C_{2}=\left(b_{1}, \ldots, b_{s}, b_{1}\right)$ and that $\left\{a_{1}, b_{1}\right\} \in E(C)$. Moreover, if $v \in V(w)$ and $v \notin N_{G}(c) \cup\{c\}$, then $v \in\left\{a_{1}, b_{1}\right\}$ because $b\left(C \backslash\left\{a_{j}\right\}\right)=b\left(C \backslash\left\{b_{j}\right\}\right)=0$ for all $j \geq 2$. Therefore we can suppose that $a_{1} \in V(w)$ and $a_{1} \notin N_{G}(c)$; thus $b\left(G \backslash\left\{a_{1}\right\}\right)=0$ and $b\left(C \backslash\left\{a_{1}\right\}\right)=1$. Since $b\left(C \backslash\left\{a_{1}\right\}\right)=1$, we can assume that $E(C)=E\left(C_{1}\right) \cup E\left(C_{2}\right) \cup\left\{\left\{a_{1}, b_{1}\right\},\left\{a_{1}, b_{j_{2}}\right\}, \ldots,\left\{a_{1}, b_{j_{k}}\right\}\right\}$ for some $k \geq 1$, and $j_{2}, \ldots, j_{k}$ are odd because $C$ is an odd partial band. Moreover, since $b\left(G \backslash\left\{a_{1}\right\}\right)=0$ it follows that $N_{G}(c)=\left\{a_{i}, b_{j}\right\}$ for some $1<i \leq r, 1 \leq j \leq s$ or $N_{G}(c)=\left\{a_{i}, a_{j}\right\}$ for some $1<i<j \leq r$ and $i \not \equiv j(\bmod 2)$. If $N_{G}(c)=\left\{a_{i}, a_{j}\right\}$, then $b_{1} \notin V(w)$ because $b\left(G \backslash\left\{b_{1}\right\}\right)=b\left(C \backslash\left\{b_{1}\right\}\right)$. Additionally, if $N_{G}(c)=\left\{a_{i}, b_{j}\right\}$ for some $1<i \leq r, 1 \leq j \leq s$, since $G$ can not be 2-connected by Lemma 6.2. we get that $j=1$ and $\left\{a_{1}, b_{1}\right\}$ is the only edge connecting $C_{1}$ and $C_{2}$. Putting all together, we can assume that one of these occurs:

(a) $V(w)=\left\{c, a_{1}, a_{i}, b_{1}\right\}$, where $N_{G}(c)=\left\{a_{i}, b_{1}\right\}$ with $1<i \leq r$ and $E(C)=E\left(C_{1}\right) \cup E\left(C_{2}\right) \cup\left\{\left\{a_{1}, b_{1}\right\}\right\}$, or

(b) $V(w)=\left\{c, a_{1}, a_{i}, a_{j}\right\}$, where $N_{G}(c)=\left\{a_{i}, a_{j}\right\}$ with $1<i<j \leq r, i \not \equiv j(\bmod 2)$ and $E(C)=E\left(C_{1}\right) \cup E\left(C_{2}\right) \cup$ $\left\{\left\{a_{1}, b_{1}\right\},\left\{a_{1}, b_{j_{2}}\right\}, \ldots,\left\{a_{1}, b_{j_{k}}\right\}\right\}$ for some $k \geq 1$, and $j_{2}, \ldots, j_{k}$ are odd.

In both cases $w$ is a length 4 cycle by Lemma 3.1. In (a) we have that $w=\left(c, a_{i}, a_{1}, b_{1}\right)$, and we can assume that $i=2$. We proved in Proposition 6.4 that $P_{C}=\left(B_{w^{\prime}}\right)$ where $w^{\prime}=\left(a_{1}, \ldots, a_{r}, a_{1}, b_{1}, \ldots, b_{s}, b_{1}, a_{1}\right)$; thus $P_{G}=\left(B_{w}, B_{w^{\prime}}\right)$. However this is not possible because denoting $e_{1}$ and $e_{2}$ the edges $\left\{a_{1}, b_{1}\right\}$ and $\left\{a_{1}, a_{2}\right\}$, then $\left(B_{w}, B_{w^{\prime}}\right) \subsetneq J:=\left(x_{1}, x_{2}\right)$ and $2=\operatorname{ht}\left(P_{G}\right)<\operatorname{ht}(J)=2$. Finally, if (b) occurs we have that $w=\left(c, a_{i}, a_{1}, a_{j}\right)$, which implies that $i=2, j=r$. Therefore $G$ is a CI-vertex-band.

$(\Leftarrow)$ If $\operatorname{deg}_{G}(c)=1, G$ is a complete intersection if and only if so is $C$ and $C$ is an odd partial band, which is a complete intersection by Proposition 6.4 .

If $G$ is a CI-vertex-band with $V(G)=V(C) \cup\{c\}$, where $C$ consists of two odd vertex disjoint cycles $C_{1}=$ $\left(a_{1}, \ldots, a_{r}, a_{1}\right)$ and $C_{2}=\left(b_{1}, \ldots, b_{s}, b_{1}\right)$ and $E(G)=E\left(C_{1}\right) \cup E\left(C_{2}\right) \cup\left\{\left\{a_{1}, b_{i_{1}}\right\}, \ldots,\left\{a_{1}, b_{i_{k}}\right\},\left\{c, a_{2}\right\},\left\{c, a_{r}\right\}\right\}$ where $1=b_{i_{1}}<\cdots<b_{i_{k}}$ and $b_{i_{1}}, \ldots, b_{i_{k}}$ are odd. Let us prove that $P_{G}=P_{C} \cdot k\left[x_{1}, \ldots, x_{n}\right]+\left(B_{w}\right)$ where $w=\left(c, a_{r}, a_{1}, a_{2}, c\right)$.

We have that $\operatorname{ht}\left(P_{G}\right)=k+1$, we set $e_{j}:=\left\{a_{1}, b_{i_{j}}\right\}$ for $1 \leq i \leq k, e_{k+j}:=\left\{a_{j}, a_{j+1}\right\}$ for $1 \leq j<r, e_{k+r}:=\left\{a_{1}, a_{r}\right\}$, $e_{k+r+j}:=\left\{b_{j}, b_{j+1}\right\}$ for $1 \leq j<s$ and $e_{k+r+s}:=\left\{b_{1}, b_{s}\right\}, e_{k+r+s+1}:=\left\{c, a_{2}\right\}$ and $e_{k+r+s+2}:=\left\{c, a_{r}\right\}$.

For every $j \in\{1, \ldots, k-1\}$, let $w_{j}$ be the even primitive cycle

$$
w_{j}:=\left(b_{i_{j}}, a_{1}, b_{i_{j+1}}, b_{i_{j+1}-1}, \ldots, b_{i_{j}}\right)
$$

and $w_{k}:=\left(b_{i_{k}}, a_{1}, C_{1}, a_{1}, b_{1}, b_{s}, \ldots, b_{i_{k}}\right)$.

If we denote $B_{w_{j}}=x^{\alpha_{j}}-x^{\beta_{j}}$ with $\alpha_{j}, \beta_{j} \in \mathbb{N}^{k+r+s+2}$ for every $j \in\{1, \ldots, k\}$ and we let $B$ be the $k \times(k+r+s+2)$ matrix whose $j$-th row is $\alpha_{j}-\beta_{j}$; then we proved in Proposition 6.4 that $B$ is dominating and $\Delta_{k}(B)=1$.

We denote by $B^{\prime}$ the matrix obtained by adding to $B$ the row $e_{k+1}-e_{k+r}-e_{k+r+s+1}+e_{k+r+s+2} \in \mathbb{Z}^{k+r+s+2}$. Then $\Delta_{k+1}\left(B^{\prime}\right)=1$ because $\Delta_{k}(B)=1$, if we prove that $B^{\prime}$ is dominating then $P_{G}=\left(B_{w_{1}}, \ldots, B_{w_{k}}, B_{w}\right)$ and it is a complete intersection. Since the columns $k+r+s+1$ and $k+r+s+2$ have only one nonzero entry, if we denote by $B^{\prime \prime}$ the matrix obtained by removing these two columns from $B^{\prime}$, by Lemma 2.5 we get that $B^{\prime}$ is dominating if and only if so is $B^{\prime \prime}$. Assume that $B^{\prime \prime}$ has a mixed square submatrix $D$, since $B$ is dominating then the last row of $B^{\prime \prime}$ is in $D$, moreover the columns $k+1$ and $k+r$ of $B^{\prime \prime}$ have to be in $D$ because these are the only two nonzero entries in the last row of $B^{\prime \prime}$. Furthermore the columns $k+1$ and $k+r$ of $B^{\prime \prime}$ have only two nonzero entries, which are those in the rows $k$ and $k+1$ and the entries in the row $k$ are both negative. So, if we remove the last row of $D$ and the column $k+1$ we get $D^{\prime}$ another square matrix of $B^{\prime \prime}$ which is mixed; but $D^{\prime}$ is also a submatrix of $B$; which it is a contradiction. 
Lemma 6.17. Let $G$ be a connected graph with $V(G)=V(C) \cup\left\{c_{1}, c_{2}\right\}$ where $C$ is an odd partial band, $\left\{c_{1}, c_{2}\right\} \in E(G)$ and $c_{1}, c_{2}$ have degree $\geq 2$. Then, $G$ is a complete intersection if and only if $G$ is a 2-clique-sum of $C$ and a length 4 cycle $C$.

Proof. $(\Rightarrow)$ For $i=1,2$ we have that $G_{i}:=G \backslash\left\{c_{i}\right\}$ is a complete intersection, then by Lemma6.16 either deg $\operatorname{deg}_{G_{i}}\left(c_{3-i}\right)=$ 1 or $G_{i}$ is a CI-vertex-band. Firstly assume that $G_{1}$ and $G_{2}$ are CI-vertex-bands and denote $C_{1}=\left(a_{1}, \ldots, a_{r}, a_{1}\right)$ and $C_{2}=\left(b_{1}, \ldots, b_{s}, b_{1}\right)$ with $\left\{a_{1}, b_{1}\right\} \in E(G)$ the two odd primitive cycles such that $V(C)=V\left(C_{1}\right) \cup V\left(C_{2}\right)$. If $\left\{c_{i}, a_{j}\right\} \in E(G)$ for $i=1,2, j=2, r$, then $G$ has a subgraph $\mathcal{K}_{2,3}$ with vertices $\left\{c_{1}, c_{2}, a_{1}, a_{2}, a_{r}\right\}$ and this is not possible. If one has that $\left\{c_{1}, a_{r}\right\},\left\{c_{1}, a_{2}\right\},\left\{c_{2}, b_{s}\right\},\left\{c_{2}, b_{2}\right\} \in E(G)$, then there are three even paths connecting $c_{1}$ and $a_{1}$, namely $\mathcal{P}_{1}:=\left(c_{1}, a_{2}, a_{1}\right), \mathcal{P}_{2}:=\left(c_{1}, a_{r}, a_{1}\right)$ and $\mathcal{P}_{3}:=\left(c_{1}, c_{2}, b_{2}, b_{1}, a_{1}\right)$ and this is not possible by Theorem 5.7 So assume now that $\operatorname{deg}_{G}\left(c_{1}\right)=2$ and $G_{1}$ is a CI-vertex-band; then $P_{G}=P_{G_{1}} \cdot k\left[x_{1}, \ldots, x_{n}\right]+\left(B_{w}\right)$ where $w$ is an even closed walk with $V(w)=\left\{c_{1}\right\} \cup N_{G}\left(c_{1}\right) \cup\left\{u \in V(G) \mid b(G \backslash\{u\})<b\left(G \backslash\left\{u, c_{1}\right\}\right)\right\}$. Since $c_{1}, c_{2} \in V(w)$, we get that $a_{2}$ or $a_{r} \in V(w)$, say $a_{2} \in V(w)$. But $b\left(G \backslash\left\{c_{1}, a_{2}\right\}\right)=0$, so $a_{2} \in N_{G}\left(c_{1}\right)$ and $V(w)=\left\{c_{1}, c_{2}, a_{2}, b_{1}\right\}$, but such a closed walk does not exist.

Then we have proved that $\operatorname{deg}_{G}\left(c_{1}\right)=\operatorname{deg}_{G}\left(c_{2}\right)=2$ and there exist $u_{1}, u_{2} \in V(C)$ such that $\left\{c_{1}, u_{1}\right\},\left\{c_{2}, u_{2}\right\} \in E(G)$. Then by Theorem 4.8, $P_{G}=P_{G \backslash\left\{c_{2}\right\}} \cdot k\left[x_{1}, \ldots, x_{n}\right]+\left(B_{w}\right)$ where $w$ is an even closed walk with $V(w)=\left\{c_{2}\right\} \cup N_{G}\left(c_{2}\right) \cup\{u \in$ $\left.V(G) \mid b(G \backslash\{u\})<b\left(G \backslash\left\{u, c_{2}\right\}\right)\right\}$. Then, $w=\left(u_{1}, c_{1}, c_{2}, u_{2}, v_{1}, \ldots, v_{t}, u_{1}\right)$ for some $t \geq 0$ and $b\left(G \backslash\left\{v_{i}\right\}\right)<b\left(G \backslash\left\{v_{i}, c_{2}\right\}\right)$ for $1 \leq i \leq t$. Nevertheless, $b\left(G \backslash\left\{z, c_{2}\right\}\right) \geq 1$ if and only if $z=u_{1}$ or $z=a_{1}$ and $\operatorname{deg}_{C}\left(a_{i}\right)=2$ for every $i \geq 2$ or $z=b_{1}$ and $\operatorname{deg}_{C}\left(b_{i}\right)=2$ for every $i \geq 2$. Then $\left\{c_{1}, c_{2}, u_{1}, u_{2}\right\} \subset V(w) \subset\left\{c_{1}, c_{2}, u_{1}, u_{2}, a_{1}, b_{1}\right\}$. If $|V(w)|=6$, then we can assume that $u_{1}=a_{2}, u_{2}=b_{2}$ and $\left\{a_{1}, b_{1}\right\}$ is the only edge connecting $C_{1}$ and $C_{2}$ but this contradicts Lemma 6.2. If $|V(w)|=5$, then $w$ is not an even cycle and by Lemma 3.1, $u_{1}=u_{2}$; however, if $u_{1} \in V\left(C_{1}\right)$, then $b\left(G \backslash\left\{b_{1}, c_{2}\right\}\right)=b\left(G \backslash\left\{b_{1}\right\}\right)$ and $b_{1} \notin V(w)$, which is a contradiction. Then $|V(w)|=4, w$ is a cycle and $\left\{u_{1}, u_{2}\right\} \in E(G)$; so $G$ is a 2-clique-sum of $C$ and the length 4 cycle $\left(c_{1}, c_{2}, u_{2}, u_{1}, c_{1}\right)$.

$(\Leftarrow)$ It follows directly from Lemma 6.10 and Proposition 6.4

Now we can state and prove the following characterization theorem, which allows us to list all families of complete intersection graphs $G=[C ; R]$ such that $R$ is 2-connected and $C$ is connected.

Theorem 6.18. Let $G=[C ; R]$ be a connected graph. If $R$ is 2-connected and $C$ is connected, then $G$ is a complete intersection if and only if $G$ is one of the following graphs:

(a) a bipartite ring graph,

(b) a 1-clique-sum of a bipartite ring graph and either

(b.1) a CI-odd-partial-wheel,

(b.2) a 1-clique-sum of an odd partial band and an edge, or

(b.3) a CI-vertex-band,

(c) a 2-clique-sum of a bipartite ring graph and either

(c.1) a CI-double-wheel, or

(c.2) an odd partial band.

Proof. $(\Rightarrow$ ) Since $G$ is connected, by Theorem 6.5 we have that $R$ is a bipartite ring graph and $C$ is either the empty graph, an odd primitive cycle or an odd partial band. If $C$ is the empty graph, then $G=R$ is a bipartite ring graph. Otherwise, by Proposition 6.8 either

(1) there exists a $b_{1} \in V(R)$ such that $\left[V(C) \cup\left\{b_{1}\right\}\right]$ is a complete intersection, or 
(2) there exist two adjacent vertices $b_{1}, b_{2} \in V(R)$ such that $\left[V(C) \cup\left\{b_{1}, b_{2}\right\}\right]$ is a complete intersection.

Assume (1) holds, if $C$ is an odd primitive cycle, by Lemma6.12 we obtain (b.1), and if $C$ is an odd partial band, by Lemma 6.16 we obtain (b.2) or (b.3). Assume now that (2) holds, if $C$ is an odd primitive cycle, by Lemma 6.14 we have (c.1), and if $C$ is an odd partial band, by Lemma 6.17 we have (c.2).

$(\Leftarrow)$ It follows from Lemmas $6.10,6.12,6.14,6.16$ and 6.17

Simis, Vasconcelos and Villarreal [26] characterized the normality of $k[G]$ in the following way.

Theorem 6.19. If $G$ is connected, then $k[G]$ is normal if and only if every two vertex disjoint odd cycles are connected by an edge.

From this description, one deduces that if a graph $G=[C ; R]$ verifies that $k[G]$ is normal, then $C$ is connected. Moreover, $G$ cannot have a CI-vertex-band as induced subgraph.

Thus, we can conclude the following results, which are the normal versions of Theorem 6.5 and Theorem 6.18

Corollary 6.20. Let $G=[C ; R]$ be a connected graph such that $k[G]$ is normal. If $G$ is a complete intersection, then

- $R$ is a bipartite ring graph, and

- $C$ is either the empty graph, an odd primitive cycle or an odd partial band.

Corollary 6.21. Let $G=[C ; R]$ be a connected graph such that $k[G]$ normal. If $R$ is 2-connected, then $G$ is a complete intersection if and only if $G$ is one of the following graphs:

(a) a bipartite ring graph,

(b) a 1-clique-sum of a bipartite ring graph and either

(b.1) a CI-odd-partial-wheel, or

(b.2) a 1-clique-sum of an odd partial band and an edge,

(c) a 2-clique-sum of a bipartite ring graph and either

(c.1) a CI-double-wheel, or

(c.2) an odd partial band.

\section{References}

[1] S.S. Abhyankar, Algebraic Geometry for Scientists and Engineers, Mathematical Surveys and Monographs 35, American Mathematical Society (1990).

[2] I. Bermejo, I. García-Marco, J.J. Salazar-González, An algorithm for checking whether the toric ideal of an affine monomial curve is a complete intersection, J. Symb. Comput. 42, 971-991 (2007).

[3] I. Bermejo, I. García-Marco, Complete intersections in simplicial toric varieties, J. Symb. Comput. (2014), to appear.

[4] R. Diestel, Graph Theory, Graduate Texts in Mathematics 173, Springer-Verlag, New York, 2nd Ed. (2000).

[5] L. Doering, T. Gunston, Algebras arising from planar bipartite graphs, Comm. Algebra 24, 3589-3598 (1996).

[6] K. Fischer, W. Morris, J. Shapiro, Affine semigroup rings that are complete intersections. Proc. Amer. Math. Soc. 125 (11), 3137-3145 (1997).

[7] K. Fischer, W. Morris, J. Shapiro, Mixed dominating matrices, Linear Algebra Appl. 270, 191-214 (1998).

[8] K. Fischer, J. Shapiro, Mixed matrices and binomial ideals, J. Pure Appl. Algebra 113, 39-54 (1996). 
[9] I. Gitler, E. Reyes, J.A. Vega, Complete intersection toric ideals of oriented graphs and chorded-theta subgraphs, J. Algebraic Combin. 38 (3), 721-744 (2013).

[10] I. Gitler, E. Reyes, R.H. Villarreal, Ring graphs and toric ideals, Electronic Notes in Discrete Mathematics 28C, 393-400 (2007).

[11] I. Gitler, E. Reyes, R.H. Villarreal, Ring graphs and complete intersection toric ideals, Discrete Math. 310 (3), $430-441$ (2010).

[12] I. Gitler, C. Valencia, Multiplicities of edge subrings, Discrete Math. 302, 107-123 (2005).

[13] F. Harary, Graph Theory, Addison-Wesley, Reading, MA (1972).

[14] J. Herzog, Generators and relations of abelian semigroups and semigroup rings, Manuscripta Math. 3, 175-193 (1970).

[15] S. Hosten, J. Shapiro, Primary Decomposition of Lattice Basis Ideals, J. Symb. Comput. 29 (4-5), 625-639 (2000).

[16] A. Katsabekis, M. Morales, A. Thoma, Binomial generation of the radical of a toric ideal, J. Algebra 324 (6), 1334-1346 (2010).

[17] M. Katzman, Bipartite graphs whose edge algebras are complete intersections, J. Algebra 220, 519-530 (1999).

[18] A.S. LaPaugh, C.H. Papadimitriou, The even path problem for graphs and digraphs, Networks 14, 507-513 (1984).

[19] H. Matsumura, Commutative Algebra, 2nd edition, The Benjaming/Cummings publishing company (1980).

[20] M. Morales, A. Thoma, Complete intersection lattice ideals, J. Algebra 284, 755-770 (2005).

[21] H. Ohsugi, T. Hibi, Toric ideals generated by quadratic binomials, J. Algebra 218, 509-527 (1999).

[22] E. Reyes, Complete intersection toric ideals of oriented graphs, Morfismos 9 (2), 71-82 (2005).

[23] E. Reyes, C. Tatakis, A. Thoma, Minimal generators of toric ideals of graphs, Adv. in Appl. Math. 48 (1), 64-78 (2012).

[24] G. Scheja, O. Scheja and U. Storch, On regular sequences of binomials, Manuscripta Math. 98, 115-132 (1999).

[25] A. Simis, On the Jacobian module associated to a graph, Proc. Amer. Math. Soc. 126, 989-997 (1998).

[26] A. Simis, W.V. Vasconcelos, R.H. Villarreal, The integral closure of subrings associated to graphs, J. Algebra 199, 281-289 (1998).

[27] B. Sturmfels, Gröbner Bases and Convex Polytopes, University Lecture Series 8, American Mathematical Society, Rhode Island (1996).

[28] C. Tatakis, A. Thoma, On complete intersection toric ideals of graphs, J. Algebr. Comb. 38 (2), 351-370 (2013).

[29] R.H. Villarreal, Rees algebras of edge ideals, Comm. Algebra 23, 3513-3524 (1995).

[30] R.H. Villarreal, Monomial Algebras, Marcel Dekker, New York (2001).

[31] R.H. Villarreal, Combinatorial Optimization Methods in Commutative Algebra, in progress. 